\title{
REVIEWS \\ Theoretical Study of Molecular Association and Thermoreversible Gelation in Polymers
}

\author{
Fumihiko TANAKA \\ Department of Polymer Chemistry, Graduate School of Engineering, Kyoto University, Kyoto 606-8501, Japan
}

(Received April 1, 2002; Accepted May 7, 2002)

\begin{abstract}
This paper reviews our recent theoretical studies of molecular association and thermoreversible gelation in polymer solutions and blends. We first classify fundamental types of association, and propose their static and dynamic characterization. We then develope general theory of associating polymers to study phase transitions induced by molecular association. These transitions include macro- and microphase separation, micellization, hydration, thermoreversible gelation and liquid-crystalization. As for the origin of associative forces, we focus on hydrogen bonding and hydrophobic aggregation. Detailed study on thermoreversible gelation with multiple cross-link junctions is presented. Paying special attention to the multiplicity and sequence length of the network junctions, we derive phase diagrams with coexisting gelation and phase separation, and compare them with experimental data. Local and global structures of the gel networks are studied from molecular point of view. The theory is applied to more complex thermoreversible gels such as binary networks (interpenetrating networks, alternating networks and randomely mixed networks), hydrated networks with high-temperature gelation, gelation strongly coupled to polymer conformational transitions such as coil-to-helix transition. To study dynamics of thermoreversible gels, a simple transient network model is introduced, and creation and annihilation of junctions in the networks are theoretically described. Stationary non-linear viscosity and the dynamic mechanical moduli are calculated as functions of the shear rate, frequency and the chain disengagement rate. From the peak of the loss modulus, the lifetime $\tau_{\times}$of the junction is estimated, and from the high frequency plateau of the storage modulus, the number of elastically effective chains in the network is found. Transient phenomena such as stress relaxation and stress overshoot are also theoretically studied. Results are compared with the recent experimental reports on the rheological study of hydrophobically modified water-soluble polymers.

KEY WORDS Associating Polymers / Thermoreversible Gelation / Junction Multiplicity / Multicriticality / Transient Networks / Shear Thickening / Elastically Effective Chains /
\end{abstract}

This paper reviews our recent theoretical studies of molecular association and thermoreversible gelation in polymer solutions. We introduce new theoretical frameworks to study phase transitions induced by association of polymer chains and dynamics of aggregation/dissociation processes.

In most polymer blends or solutions, polymer chains carry active groups interacting with each other via strongly associative forces which are capable of forming temporal bonds. Hence description of the system in terms of van der Waals type contact energy - or $\chi$-parameter in lattice theoretical terminology - is insufficient. These associative interactions include hydrogen bonding, ionic aggregation, electrostatic interaction, micro-crystallite formation, stereocomplex formation, solvent-complex formation, etc. Since the binding energy is of the order of thermal energy, bond formation is reversible; bonds are created and destroyed by the change in temperature and concentration. Extensive polydispersity inherent to such associating solutions is therefore thermally controlled. The spatial and temporal organizations in polymeric materials formed by such associative forces are one of the un- investigated important subjects in the polymer science yet to be explored. Typical examples of these organizations are (hetero-)dimerization, solvation, micellization, thermoreversible gelation, liquid-crystallization and various mesophase formation. Consideration of such associative interactions superimposed on the familiar description of statistical chains can lead to the application of the polymer science in numerous systems of practical importance. Polymers interacting by associative forces also exhibit characteristic rheological properties such as shear thickening, stress overshoot, etc. so that they imply vast industrial applications as rheology control agents.

The strength of association is generally described by the association constant defined by

$$
\lambda(T) \equiv \exp \left(-\beta \Delta f_{0}\right)
$$

where $\beta \equiv 1 / k_{\mathrm{B}} T$ is the reciprocal temperature, and $\Delta f_{0}$ the standard free energy change on binding a single associative group into an aggregate, or a network junction. If the associative group (or chain block) consists of $\zeta$ statistical segments, as in aggregation of hydrophobic short chains, the free energy change can be written 
as

$$
\Delta f_{0}=\zeta(\Delta h-T \Delta s)
$$

by the use of the binding enthalpy $\Delta h$ and entropy $\Delta s$ per statistical unit. The number $\zeta$ is called the sequence length of a junction.

Another important structural parameter of a junction is its multiplicity. The multiplicity $k$ is defined by the number of groups combined together in a single aggregate. A cross-link by covalent bond has multiplicity $k=2$ because of the pairwise bonding. However, since most thermoreversible gels have multiple crosslinks, ${ }^{1-5}$ multiplicity is an important concept in studying association of polymer chains.

From a dynamic point of view, the time scale of reorganization in the network junctions is characterized by the average duration time $\tau_{\times}$for an associative group to be in a bound state. It is governed by the free energy barrier $\Delta F^{\dagger}$ separating the bound state from the free one:

$$
\tau_{\times}=\tau_{0} \exp \left(\beta \Delta F^{\dagger}\right) .
$$

( $\tau_{0}$ being a microscopic time of monomer motion.) The rheological time scale governing the dynamics of transient networks can therefore be adjusted by this barrier height in the associative interaction.

Paying special attention to such important structural and dynamic parameters, we first introduce equilibrium statistical-mechanical theory of associating polymer solutions, and then examine the results of its application to specific important problems by focusing on thermoreversible gelation. We then move onto dynamics of transient polymer networks. We introduce molecular picture for studying rheological properties of thermoreversible gels, calculate stationary and transient flow properties, and compare with experiments.

\section{THERMODYNAMIC THEORY OF ASSOCIATING POLYMERS}

\section{Model Associating Polymers}

\section{Stoichiometric Definition of the Mixture}

Consider a binary mixture of linear polymers A and $B$. The number of statistical units on a chain is assumed to be $n_{\mathrm{A}}$ for an A chain and $n_{\mathrm{B}}$ for a B chain. It is also assumed that each chain carries a fixed number $f$ (for A chain) and $g$ (for B chain) of reactive groups capable of forming reversible bonds. In thermal equilibrium, forces working among associative groups form intermolecular clusters with a wide range of aggregation number. If either of the functionalities exceeds three, a cluster grows to macroscopic dimensions as soon as a threshold in temperature or in composition is reached. Above the threshold a three-dimensional network, most generally comprized of the two components, is formed. ${ }^{1-5}$ To describe network formation, we start from a lattice theoretical picture. ${ }^{6-9}$

Let us first divide the total volume $V$ of the system into small cells of size $a$ of the monomeric units on a chain. ${ }^{6}$ We then have total number $\Omega \equiv V / a^{3}$ of the microscopic cells. We first specify the part of the system containing only finite-size clusters, which will be referred to as sol. Let $N_{l, m}$ be the number of $(l, m)$ clusters consisting of $l \mathrm{~A}$-chains and $m \mathrm{~B}$-chains. The total volume fraction of A-chains in the sol is given by

$$
\phi_{\mathrm{A}}^{\mathrm{S}}=n_{\mathrm{A}} \sum_{l, m} l v_{l, m},
$$

where $v_{l, m} \equiv N_{l, m} / \Omega$ is the number of clusters (per lattice cell). Similarly, the total volume fraction of Bchains in the sol is given by

$$
\phi_{\mathrm{B}}^{\mathrm{S}}=n_{\mathrm{B}} \sum_{l, m} m v_{l, m} .
$$

The total volume fraction of the sol in the system is given by $\phi^{\mathrm{S}}=\phi_{\mathrm{A}}^{\mathrm{S}}+\phi_{\mathrm{B}}^{\mathrm{S}}$. This should be equal to unity for nongelling systems, or pregel regime of gelling systems, but can become smaller than unity as soon as an infinite network - referred to as gel - appears, i.e., in the postgel regime. In the postgel regime, the volume fraction of $i$ chains in the gel network is given by $\phi_{i}^{\mathrm{G}}=\phi_{i}-\phi_{i}^{\mathrm{S}}$ for $i=\mathrm{A}$ and $\mathrm{B}$, where $\phi_{i}$ is the experimentally controlled volume fraction (or composition) of the species $i$. Since we have an identity $\phi_{\mathrm{A}}+\phi_{\mathrm{B}}=1$, we can take $\phi_{\mathrm{A}}$ as an independent variable, and write it simply as $\phi$. The volume fraction of B is then given by $\phi_{\mathrm{B}}=1-\phi$.

\section{Free Energy of the Mixture}

In order to study thermodynamic properties, we start from the standard reference state in which unconnected A-chains and B-chains are prepared separately in a hypothetical crystalline state. ${ }^{6,8}$ We first consider the free energy change $\Delta F_{\text {rea }}$ to bring the system to a fictitious intermediate state in which the chains are disoriented and connected in such a way that the cluster distribution is exactly the same as the real one. ${ }^{12,13,19}$ It is given by

$$
\beta \Delta F_{\text {rea }} / \Omega=\sum_{l, m} \Delta_{l, m} v_{l, m}+\delta_{\mathrm{A}} v_{\mathrm{A}}^{\mathrm{G}}+\delta_{\mathrm{B}} v_{\mathrm{B}}^{\mathrm{G}},
$$

where $\Delta_{l, m}$ is the free energy produced when a single $(l, m)$ cluster is formed from $l \mathrm{~A}$-chains and $m$ B-chains. Let $\mu_{l, m}^{\circ}$ be the internal free energy of an $(l, m)$ cluster. The free energy difference $\Delta_{l, m}$ is then given by

$$
\Delta_{l, m}=\beta\left(\mu_{l, m}^{\circ}-l \mu_{1,0}^{\circ}-m \mu_{0,1}^{\circ}\right) .
$$


Under a constant pressure, $\mu_{l, m}^{\circ}$ is equivalent to the internal free energy produced by combination, configurational change, and bond formation of the constitutional chains. Similarly $\delta_{i}(i=\mathrm{A}, \mathrm{B})$ is the free energy change produced when an isolated chain of the species $i$ is connected to the gel network. They are given by $\delta_{\mathrm{A}}=\beta\left(\mu_{\mathrm{A}}^{\circ \mathrm{G}}-\mu_{1,0}^{\circ}\right)$, and $\delta_{\mathrm{B}}=\beta\left(\mu_{\mathrm{B}}^{\circ \mathrm{G}}-\mu_{0,1}^{\circ}\right)$, where $\mu_{i}^{\circ \mathrm{G}}$ is the chemical potential of the species $i$ in the gel network.

In the second step, we mix these clusters to get to a real mixture. According to the conventional lattice theory ${ }^{8,9}$ of polydisperse polymer mixtures, the mixing free energy $\Delta F_{\text {mix }}$ in this process is given by

$$
\beta \Delta F_{\text {mix }} / \Omega=\sum_{l, m} v_{l, m} \ln \phi_{l, m}+\chi \phi(1-\phi),
$$

where $\phi_{l, m} \equiv\left(n_{\mathrm{A}} l+n_{\mathrm{B}} m\right) v_{l, m}$ is the volume fraction of $(l, m)$-clusters, $\chi$ the Flory $\chi$-parameter which specifies the strength of the van der Waals type contact interaction between the monomers of different species. This $\chi$-parameter varies with the temperature, but is independent of the composition. The total free energy from which our theory starts is given by the sum of the above two parts:

$$
\Delta F=\Delta F_{\text {rea }}+\Delta F_{\text {mix }} .
$$

By differentiation with respect to the number $N_{l, m}$ of the clusters, we find their chemical potential $\mu_{l, m}$.

\section{Pregel Regime}

To find the equilibrium distribution of clusters, we impose multiple equilibrium conditions

$$
\Delta \mu_{l, m}=l \Delta \mu_{1,0}+m \Delta \mu_{0,1}
$$

for all possible combinations of the integers $(l, m)$. Upon substitution of the specific forms of the chemical potentials, we find ${ }^{12}$ that the volume fractions of the clusters are given by

$$
\phi_{l, m}=K_{l, m} x^{l} y^{m},
$$

where we have written as $x$ and $y$ for simplicity for the unimer concentrations $\phi_{1,0}$ and $\phi_{0,1}$. The new constant $K_{l, m}$ (called equilibrium constant) is defined by $K_{l, m} \equiv \exp \left(l+m-1-\Delta_{l, m}\right)$, which depends only on the temperature through $\Delta_{l, m}$. The total volume fraction $\phi^{S}$ and the total number $v^{S}$ of clusters in the sol is then found by the infinite sum:

$$
\phi^{\mathrm{S}}(x, y)=\sum_{l, m} K_{l, m} x^{l} y^{m} .
$$

\section{Sol/Gel Transition and Postgel Regime}

So far we have tacitly assumed that the infinite double summation in $\phi^{\mathrm{S}}$ (and hence in $v^{S}$ ) converges.

Polym. J., Vol. 34, No. 7, 2002
These are double power series with positive coefficients, so that they are monotonically increasing functions. For a solution capable of gelling, a borderline exists which separates the unit square on the $(x, y)$ plane into a convergent region and a divergent one. Exactly on the boundary line, the sol composition $\phi^{\mathrm{S}}$ takes a finite value, but it diverges outside this line. Since the radius of convergence generally depends on the composition, let us express the boundary by a parametric representation $\left(x^{*}(\phi), y^{*}(\phi)\right)$ for $0 \leq \phi \leq 1$. The value $\phi^{S}\left(x^{*}, y^{*}\right)$ can become smaller than unity for certain region of the concentration and the temperature corresponding to the postgel regime. Hence we can find the sol/gel transition line by mapping the condition $\phi^{\mathrm{S}}\left(x^{*}, y^{*}\right)=1$ onto the temperature-concentration plane.

In the postgel regime, a chain participating in the gel network must be in a chemical equilibrium with an unassociated chain of the same species. This imposes the additional conditions

$$
\Delta \mu_{1,0}=\Delta \mu_{\mathrm{A}}^{\mathrm{G}} \quad \text { and } \quad \Delta \mu_{0,1}=\Delta \mu_{\mathrm{B}}^{\mathrm{G}},
$$

we find

$$
x^{*}=\exp \left(\delta_{\mathrm{A}}-1\right) \quad \text { and } \quad y^{*}=\exp \left(\delta_{\mathrm{B}}-1\right),
$$

for the gelling component. Asterisks indicate that they refer to the quatities after the gel point.

\section{Solution Properties}

Let us now find some important physical quatities characterizing the mixture.

(1) Osmotic Pressure The osmotic pressure $\pi$ of the A component is essentially the chemical potential of the B species with opposite sign, and given by

$$
\beta \pi / n_{\mathrm{B}} a^{3}=-(1+\log y) / n_{\mathrm{B}}+v^{\mathrm{S}}(x, y)-\chi \phi^{2},
$$

in the pregel regime. In a polymer solution in which $\mathrm{B}$ component is a low molecular weight solvent $\left(n_{\mathrm{B}}=1\right)$, this definition reduces to the osmotic pressure in the conventional meaning. If we expand this pressure with $n_{\mathrm{B}}=1$ in powers of the concentration, we have the virial series

$$
\pi a^{3} / k_{\mathrm{B}} T=\phi / n_{\mathrm{A}}+A_{2} \phi^{2}+A_{3} \phi^{3}+\cdots .
$$

The second virial coefficient has a reduction $\Delta A_{2}$ from $1 / 2-\chi$ due to the associative interaction.

(2) Phase Separation The two-phase equilibrium conditions, or a binodal lines can be found by balancing the chemical potential of each component: $: 8,9$

$$
\begin{aligned}
& \Delta \mu_{\mathrm{A}}\left(\phi^{\prime}, T\right)=\Delta \mu_{\mathrm{A}}\left(\phi^{\prime \prime}, T\right), \\
& \Delta \mu_{\mathrm{B}}\left(\phi^{\prime}, T\right)=\Delta \mu_{\mathrm{B}}\left(\phi^{\prime \prime}, T\right),
\end{aligned}
$$


where $\phi^{\prime}$ and $\phi^{\prime \prime}$ are the composition of the dilute A phase and concentrated A phase respectively. If the concentrated phase lies inside the postgel regime, the r.h.s. of these equations must be replaced by $\Delta \mu_{\mathrm{A}}^{*}\left(\phi^{\prime \prime}, T\right)$ and $\Delta \mu_{\mathrm{B}}^{*}\left(\phi^{\prime \prime}, T\right)$.

(3) Stability Limit The thermodynamic stability limit or a spinodal line can be found for the binary system by a single condition $\left(\partial \Delta \mu_{\mathrm{A}} / \partial \phi\right)_{\mathrm{T}}=0$, or equivalently $\partial\left(\Delta \mu_{\mathrm{A}} / n_{\mathrm{A}}-\Delta \mu_{B} / n_{\mathrm{B}}\right) / \partial \phi=0$. Our result leads to the equation

$$
\frac{\kappa_{\mathrm{A}}(\phi)}{n_{\mathrm{A}} \phi}+\frac{\kappa_{\mathrm{B}}(\phi)}{n_{\mathrm{B}}(1-\phi)}-2 \chi=0,
$$

where the new functions are defined by

$$
\begin{aligned}
& \kappa_{\mathrm{A}}(\phi) \equiv \phi \partial \log x / \partial \phi \quad \text { and } \\
& \kappa_{\mathrm{B}}(\phi) \equiv-(1-\phi) \partial \log y / \partial \phi .
\end{aligned}
$$

For homopolymer association where only A-chains are associated, for instance, $\kappa_{\mathrm{A}}$ reduces to the reciprocal of weight-average cluster size as in the polydisperse polymer solutions. ${ }^{9}, 14$ In heteropolymer association, however, $k$ 's are related to the average cluster sizes in a more complicated ways.

\section{Microphase Formation}

In order to find the condition for the microphase formation, ${ }^{15}$ we next calculate the correlation function $S(\mathbf{q})$ of the concentration fluctuation. This function is directly measurable in the X-Ray or neutron scattering experiments as a function of the wave vector, temperature and concentration. When it is infinitely enhanced at a certain finite wave number $q$, it is the precursor of instability against the concentration fluctuation whose spatial variations have a characteric length $q^{-1}$, thus leading to the formation of an ordered state with the periodicity $q^{-1}$. In the case of block copolymers, the domain size $q^{-1}$ is of the order of the radius of gyration of a single polymer chain, and hence called a microphase separation transition (MST). From the same viewpoint, we can also find the macroscopic stability limit - or spinodal condition (SP) discussed in the preceding section - from the condition $S(q=0)=\infty$, which is equivalent to the divergence of the osmotic compressibility,

In order to obtain a specific form of the correlation function $S(\mathbf{q})$, we employ random phase approximation (RPA). ${ }^{16}$ RPA provides a classical treatment of concentration fluctuations for the incompressible mixtures of very large molecular-weight molecules. It assumes a self-consistent potential uniformly acting on all species of monomers to ensure the incompressibility condition. The detail of RPA method, as applied to our polydisperse copolymer blend, leads to

$$
S(\mathbf{q})=1 /\{G(q) / W(q)-2 \chi\},
$$

where $G(q) \equiv S_{\mathrm{AA}}^{\circ}(q)+S_{\mathrm{BB}}^{\circ}(q)+2 S_{\mathrm{AB}}^{\circ}(q)$, and $W(q) \equiv$ $S_{\mathrm{AA}}^{\circ} S_{\mathrm{BB}}^{\circ}(q)-\left[S_{\mathrm{AB}}^{\circ}(q)\right]^{2}$ are both related to the intracluster scattering functions (superscript $\circ$ shows the scattering intensity arising from a single cluster). ${ }^{16}$ For our associating blends, clusters are characterized by the set of two figures $(l, m)$, so that $S_{\mathrm{A}, \mathrm{B}}^{\circ}$ for the monomer correlation function between $\mathrm{A}$ and $\mathrm{B}$ species, for example, transforms into $S_{\mathrm{AB}}^{\circ}(q)=\sum_{l, m} C_{l m}(q) v_{l, m}$, where $C_{l m}(q)$ is the monomer correlation functions within an isolated single cluster of the type $(l, m) .{ }^{17,18}$ The divergence condition for $S(\mathbf{q})$ leads to

$$
G(q) / W(q)-2 \chi=0
$$

within the framework of RPA. If this condition is satisfied for finite $q$, the system becomes unstable against the fluctuation in the concentration whose spatial dimensions are characterized by $q^{-1}$. If it is satisfied for $q=0$ on the other hand, it is unstable against demixing into two coexistent macroscopic phases. It can be shown that the latter condition is equivalent to the eq 19.

\section{SOME IMPORTANT EXAMPLES OF NON-GELLING ASSOCIATING MIXTURES}

We first show some of the important results obtained so far for the non-gelling mixtures. In this section, we assume pairwise bonding between associative groups. The strength of the bonds can be expressed in terms of the association constants defined by

$$
\begin{aligned}
& \lambda_{\mathrm{AA}} \equiv \exp \left(-\beta \Delta f_{\mathrm{AA}}\right), \quad \lambda_{\mathrm{BB}} \equiv \exp \left(-\beta \Delta f_{\mathrm{BB}}\right), \\
& \lambda_{\mathrm{AB}} \equiv \exp \left(-\beta \Delta f_{\mathrm{AB}}\right),
\end{aligned}
$$

for three combination of pairs, ${ }^{20}$ where $\Delta f$ 's are the standard free energy changes on bonding. To prevent gelling, we assume that the functionalities of either or both species of polymers are less than or equal to two in this section.

We apply our general theory presented above, and summerize the main results in the form of phase diagrams. For the numerical calculation we introduce the reduced temperature deviation $\tau \equiv 1-\Theta / T$ from the unperturbed theta temperature $\Theta$ which is defined by the equation $\chi(\Theta)=1 / 2 n_{\mathrm{B}}$. The unrenormalized second virial coefficient of the osmotic pressure vanishes at this temperature. We then assume the Shultz-Flory form $^{8,21} \chi(T)=1 / 2-\psi_{1} \tau$ for the bare interaction parameter, where $\psi_{1}$ is a material parameter of order unity. For any one of the association constant $\lambda(T)$, 


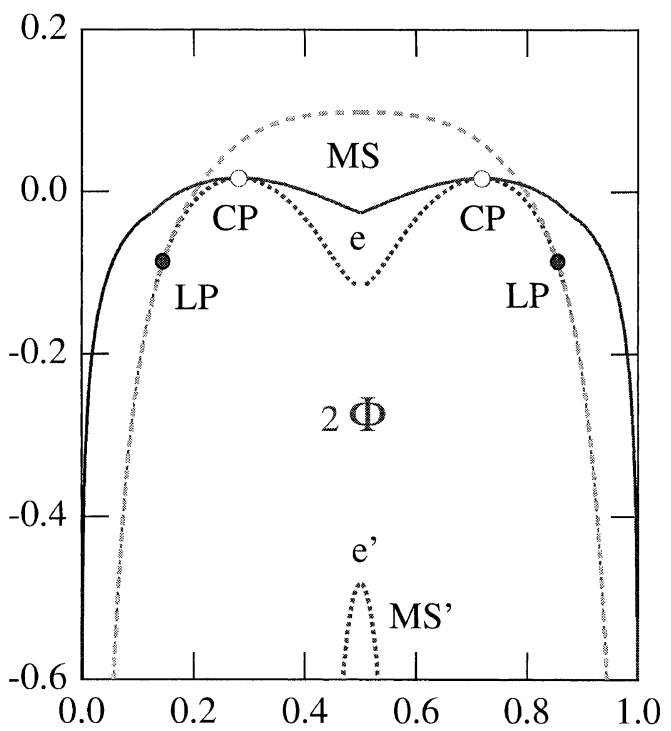

Figure 1. A typical phase diagram of associating diblock copolymers in which macro- and microphase separation compete. Binodal (solid line), spinodal (dotted line) and MST (broken line) are drawn. Critical points are indicated by CP. At the crossing of spinodal and MST line, Lifshitz points (LP) appear. At the stoichiometric composition where the number of A groups equals that of B groups, an eutectic point appears.

we rewrite as $\lambda(T)=\lambda_{0} \exp [\gamma(1-\tau)]$ with the dimensionless binding energy $\gamma \equiv \Delta \epsilon / k_{\mathrm{B}} \Theta$ and the constant $\lambda_{0} \equiv \exp \left(\Delta s / k_{\mathrm{B}}\right)$ which is related to the entropy change $\Delta s$ for bonding. ${ }^{10,11}$

\section{Dimer Formation and Side-Chain Association}

The first system we study is a mixture of A- and $\mathrm{B}$ chains in which diblock copolymers are formed by the end-to-end association (hetero-dimerization).${ }^{17}$ For such mixtures we have $f=g=1$ and $\lambda_{\mathrm{AA}}=$ $\lambda_{\mathrm{BB}}=0, \lambda_{\mathrm{AB}}>0$. A composite chain formed is a diblock copolymer A.B with a temporal junction in the middle. The system is made up of a mixture of diblock copolymers, and unassociated homopolymers of each species. It is apparently same as a mixture of chemically connected diblock copolymers dissolved in their homopolymers, ${ }^{22,23}$ but its phase behavior is in fact much richer, because the population of the block copolymers varies by the change in the temperature and the composition.

Figure 1 shows theoretical calculation of the phase diagram for a symmetric blend where both chains have the same length. ${ }^{17}$ Solid lines show the binodal, the broken lines MST, and dotted lines the spinodal. MST and spinodal meet at the two symmetric points (indicated by LP), at which the two conditions eqs 19 and 22 reduces to a single one. They are examples of the Lifshitz point, - the point where an order parameter with finite wave number starts to appear. ${ }^{24}$
The whole plane is divided into several regions, each characterized by the capital letters in it. The region with letter $\mathrm{H}$ has a homogeneously mixed fluid phase. The regions shown by MS and MS' exhibit microscopically ordered phases in which microdomains are regularly ordered. A region with the letters " $2 \Phi$ " in the figure is a biphasic region (or miscibility gap) where two distinct phases coexist. In the middle of the phase diagram we have an eutectic point (indicated by the letter $e$ ) where single microphase melt into the two coexistent homogeneously mixed fluids when the temperature is lowered. As the temperature is further lowered, we observe that the miscibility gap starts to split again at the point $e^{\prime}$ in the center of the concentration axis, and a new homogeneous microphase (shown by MS') becomes stabilized in between. The low-temperature microphase - which should be called a reentrant microphase - is stabilized simply because the population of block copolymers produced becomes so large in this low-temperature region that they homogenize the two demixed fluid phases into a single one.

Typical ordered phases experimentally confirmed recently are those induced by hydrogen bonds..$^{25-27}$ The hydrogen bond leads to thermoreversible MST if it is strong enough compared to the repulsive interaction between the polymer segments but still weak enough to break by temperature. Unfortunately, dimer formation turned out to be not strong enough. Ruokolainen and his coworkers ${ }^{28-30,32}$ have recently observed MST in the mixture of poly(4-vinyl pyridine) (P4VP) and surfactant molecules 3-pentadecyl phenol (PDP). In this sytem the hydrogen bonding between the hydroxyl group in PDP and the basic aminic nitrogen in the pyridine group leads to the formation of combshaped block copolymers with densely grafted short side chains (called molecular bottlebrush ${ }^{31}$ ). They observed a lamellar structure at low temperatures. The lamellar period $L$ was found to decrease in proportion to the reciprocal of $x$, the fraction of surfactant molecules per pyridine group in P4VP. It was also found that the MST temperature takes a minimum value (easiest MST) near the stoichiometric concentration $x=1$. Our theory can readily be extended to such side-chain associations. An example of the phase diagram for associating comb polymers is shown in Figure 2. The structures of possible mesophases inside MS region were recently studied by ten Brinke et al..$^{30,31}$

\section{Hydration in Aqueous Polymer Solutions}

The next system we consider is a polymer solution in which solvent molecules $\left(n_{\mathrm{B}}=1\right)$ attach onto the poly- 


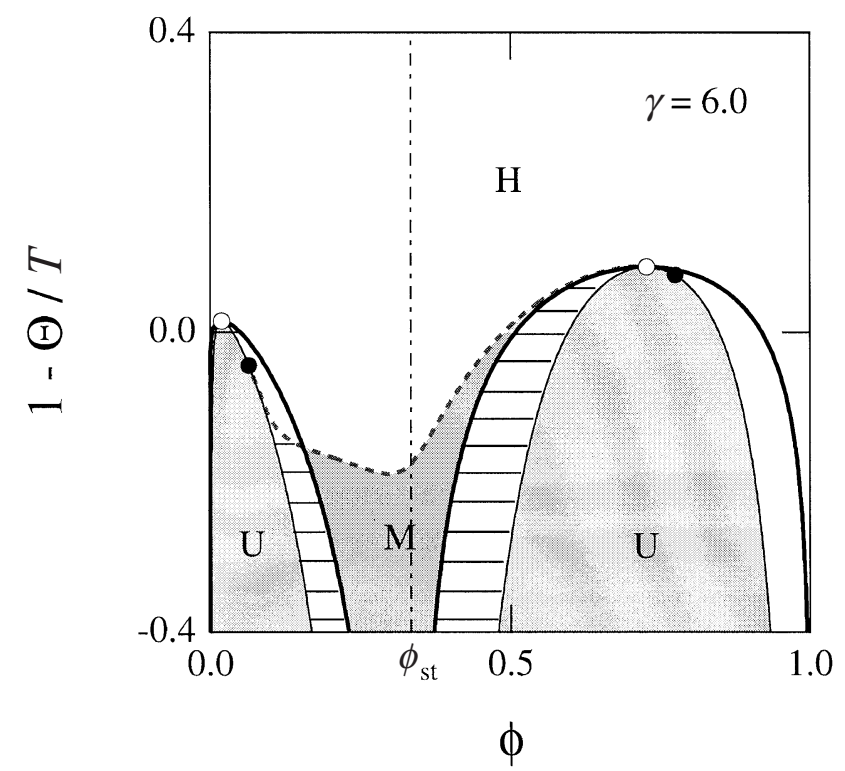

Figure 2. Phase diagram in which macro- and microphase separation compete by comb-shaped side-chain association. $n_{\mathrm{A}}=$ $1000, f=200, n_{\mathrm{B}}=10, \lambda_{0}=1.0, \gamma=6.0$. Homogeneous liquid phase $(\mathrm{H})$, microphase separated region $(\mathrm{M})$, unstable region $(\mathrm{U})$ are shown. MST is easiest at the stoichiometric composition indicated by $\phi_{s t}$. Critical solution point (white circle) and Lifshitz point (black circle) are indicated. Metastable regions inside $\mathrm{M}$ region are indicated by horizental thin lines.

mer chains $\left(n_{\mathrm{A}}=n\right)$ by reversible bonds ( $f$ large proportional to $n, g=1$, and $\left.\lambda_{\mathrm{AB}}>0, \lambda_{\mathrm{AA}}=\lambda_{\mathrm{BB}}=0\right)$. We call this phenomenon solvation. ${ }^{33}$ Hydration in aqueous polymer solutions by hydrogen bonds is the most important example. Our model predicts that the solvation can lead to the peculiar types of phase separation with closed-loop and hour-glass type miscibility gaps. ${ }^{33}$

Figure 3 shows a possible phase diagram. In this figure we fix the parameters as $\lambda_{0}=0.002$, and $\gamma=3.5$ (from the measured strength of the hydrogen bond in a solution) for a typical example. The number of the statistical unit on a polymer chain is varied from curve to curve. The number $f$ of attaching sites on a polymer chain is assumed to be proportional to the number of statistical units. The open circles show critical solution points. The solid curves show binodals, and the dashed curves spinodals. For such a small value of $\lambda_{0}$, there are two miscibility gaps for small molecular weights: one miscibility dome and one closed loop above the dome (see $n=10^{2}$ curve). The closed loop ${ }^{34-37}$ has one upper critical solution temperature (UCST) on the top and one lower critical solution temperature (LCST) on the bottom. The dome has an ordinary UCST. As the molecular weight is increased, the LCST and the UCST of the dome come closer and closer, and at certain value of $n$ (1670 in this figure) the two points merge into a higher order critical point (it is called double crit-

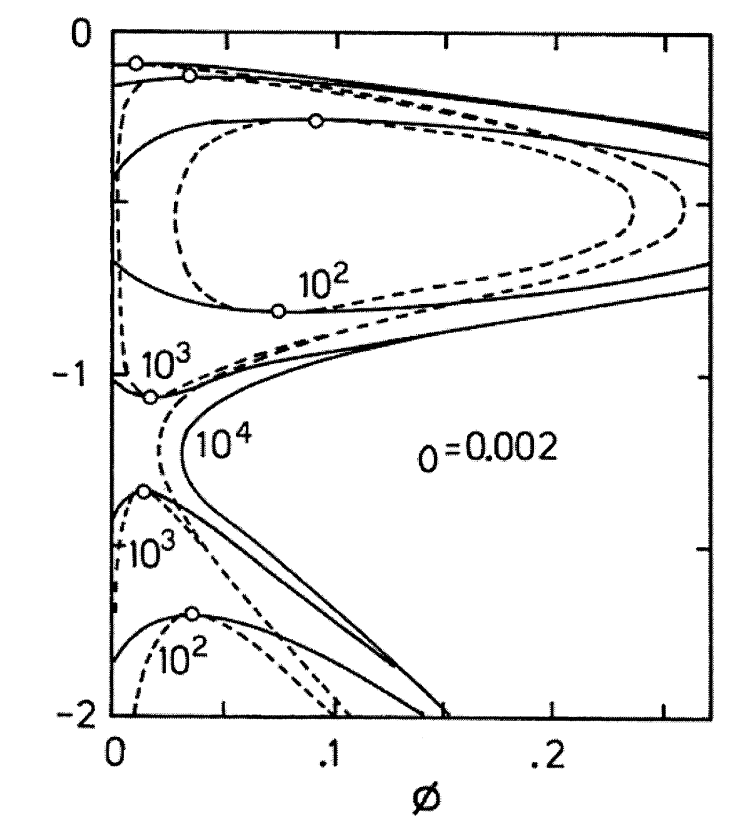

Figure 3. Phase diagram of hydrated polymer solutions. The segment number $n$ is varied from curve to curve. Binodals (solid lines) and spinodals (broken lines) are drawn. The critical solution points are indicated by white circles. LCST and UCST approaches and eventually merges into an hour-glass by increasing the polymer molecular weight. The phase separation loop vanishes by decreasing the polymer molecular weight.

ical point $).{ }^{38}$ For the molecular weights higher than this critical value, the two gaps merge into a single hourglass. On the contrary, the miscibility loop shrinks with decrease in the molecular weight, and eventually vanishes at a certain critical molecular weight ( $n=37$ for this case). This vanishing loop is called hyper critical point. For a slightly higher value of $\lambda_{0}$, however, it was found that the two gaps remain separated no matter how large the molecular weight may become. ${ }^{33}$ There are three theta temperatures under such condition to which each critical point approaches in the limit of high molecular weight. For a still larger value of $\lambda_{0}$, the closed loop gap does not appear, and we are left with an ordinary miscibility dome. Since the parameter $\lambda_{0}$ is small if the entropy loss during the bond formation is large, there must be a strong orientational or configurational constraint in the local geometry for the appearance of an hour-glass.

Figure 4 shows the comparison ${ }^{33}$ of theoretical calculation with the observed phase diagram ${ }^{39,40}$ for polyethylene oxide (PEO) in water. The number average molecular weight covers the range $2.17 \times 10^{3}-$ $1.02 \times 10^{6}$. The solid curves show the calculated binodals. The number $n$ of the statistical units on a chain is varied from curve to curve. The parameters used for fitting are: $\psi_{1}=1, \Theta=730 \mathrm{~K}, \gamma=6$, and $\lambda_{0}=$ $1.66 \times 10^{-5}$. Fitting is made mainly by adjusting the unkown parameter $\lambda_{0}$. The agreement is very good. 


\section{Poly (ethylene oxide $) / \mathrm{H}_{2} \mathrm{O}$}

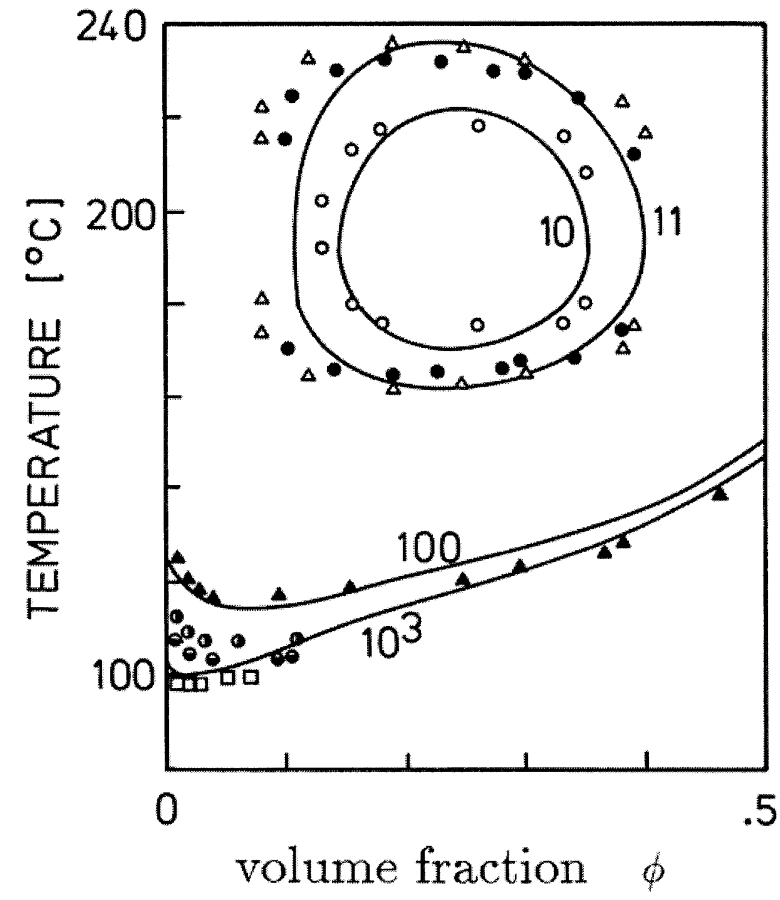

Figure 4. Phase diagram of aqueous poly(ethylene oxide) (PEO) showing closed-loop miscibility gap. Theoretical curves are fitted to the experimental data of the cloud points measured by Saeki et al. The miscibility loop expands with increase in the molecular weight. The UCST phase separation expected at low temperature cannot be seen by crystalization of PEO.

\section{Hydrogen-Bonded Supramolecules}

Some rigid molecules are known to undergo liquid crystallization when hydrogen-bonded to each other. For example, aromatic acid derivatives with alkoxy or alkyl terminal groups form dimers due to hydrogen bond between their carboxylic acid groups, and show mesomorphism. ${ }^{41-44}$ Association between different species of molecules also induces the isotropic/ anisotropic phase transition. ${ }^{27,45,46}$ Mesophases of some liquid-crystalline molecules are stabilized by hydrogen bond with different species of molecules. More markedly, some non-mesogenic molecules form a compound with a mesogenic core when hydrogen bonded. Compounds formed by hydrogen bonds are often called "supramolecule", because molecules assemble into superstructures. A supramolecule with liquid crystallinity is called "supramolecular liquid crystals". Supramolecular liquid crystals exhibit various new properties and functions. For example, they are easily switched between isotropic phase and anisotropic one by changing thermodynamic conditions. Those reported so far have various molecular architectures. For a mixture of two different species of molecules A and B, each carrying at least one rigid part that form mesogenic core by association, dimer type, trimer type, main-chain type, side-chain type, combined type, and network type are known. ${ }^{27,47}$

To describe liquid crystallization by association, we employ McMillan's free energy ${ }^{48,49}$ as $\Delta F_{\text {ori. }}$. It includes both orientational ordering of mesogenic cores and translational ordering of their center of mass. We then have

$$
\beta \Delta F_{\text {ori }}=\left\{(-\ln Z)+\frac{1}{2} \zeta\left(\eta^{2}+\alpha \sigma^{2}\right) v_{\mathrm{M}}\right\} N_{\mathrm{M}}
$$

in addition to eq 9 , where $N_{\mathrm{M}}$ is the total number of mesogenic cores in the system, and $\nu_{\mathrm{M}} \equiv N_{\mathrm{M}} / \Omega$ is the number of mesogenic cores produced by association. These depend on temperature and composition. The symbol $\eta$ expresses nematic order parameter, $\sigma$ smectic order parameter, $\zeta$ nematic interaction parameter, and $\alpha$ smectic interaction parameter. The partition function $Z$ refers to each mesogenic core and is defined by

$$
\begin{aligned}
Z(\eta, \sigma) \equiv & \frac{1}{d} \int_{0}^{d} d z \int_{0}^{1} d \cos \theta \exp \{\zeta[\eta \\
& \left.+\alpha \sigma \cos (2 \pi z / d)] P_{2}(\cos \theta) v_{\mathrm{M}}\right\},
\end{aligned}
$$

where $d$ is the distance between the neighboring planes in a smectic A structure on which the centers of mass of mesogenic cores are located. In the following it is referred to as layer thickness. The symbol $\theta$ expresses the angle between the longitudinal axis of each mesogenic core and the preferential orientational axis. The function $P_{2}(x) \equiv\left(3 x^{2}-1\right) / 2$ is the Legendre polynomial of degree 2 .

We show an example of phase diagram calculted for dimer formation. Figure 5 shows a phase diagram of a symmetric mixture with $n_{\mathrm{A}}=n_{\mathrm{B}}=10$, and $n_{\mathrm{A}}^{*}=n_{\mathrm{B}}^{*}=$ 1. Other parameters are given by $\lambda_{0}=30.0, C=0.3$, $C_{1}=-0.5$, and $C_{2}=0.05$. The inset magnifies the important part in the Figure. Thin solid line is the I/N transition line, and thick solid line the $\mathrm{N} / \mathrm{S}_{\mathrm{m}}$ transition line. Letters "I", "N", and " $\mathrm{S}_{\mathrm{m}}$ " represent the state whose free energy is lowest in each area. Dotted lines limiting the hatched metastable region are binodals. The dark gray area with "U" is the unstable region due to the new mechanism described in the preceding section, whereas the light gray area with " $U$ "' is the conventional unstable region due to demixing. White circles represent critical solution points.

At high temperature, the coexistence region caused by the I/N phase transition and that caused by repulsive interaction between the two different species of molecules appear. The mixture separates either into two I phases by the effect of mixing enthalpy or into I phase and $\mathrm{N}$ phase by the $\mathrm{I} / \mathrm{N}$ transition depending upon the composition. 

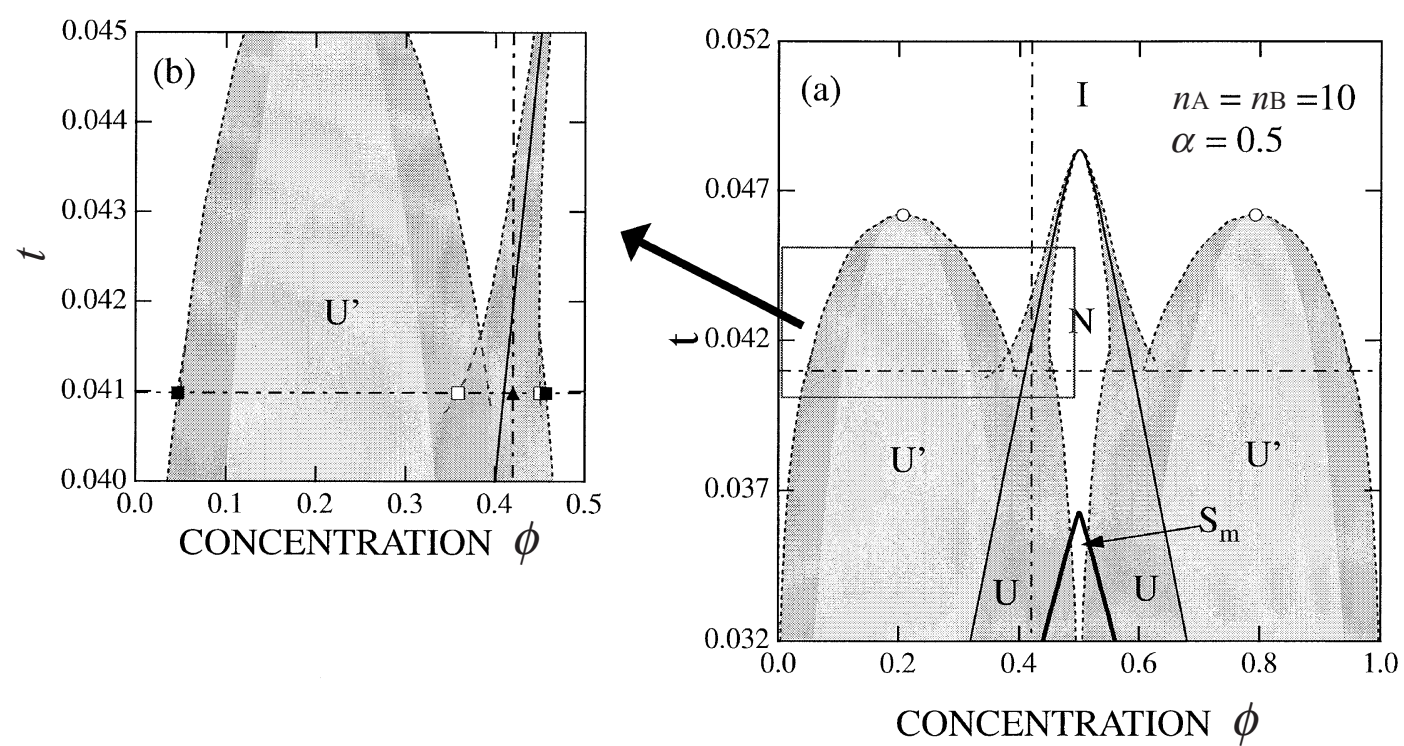

Figure 5. Phase diagram of dimer-forming hydrogen-bonded supramolecular liquid crystal, and its partial magnification. Thin solid line is the $\mathrm{I} / \mathrm{N}$ transition line, thick solid line is the $\mathrm{N} / \mathrm{S}$ transition line, and dotted line is the binodal. The hatched area is the metastable region. The dark gray area with "U" is the unstable region due to entropy difference between two different species of N structures. The light gray area with " $\mathrm{U}$ "' is the unstable region due to mixing two different species of molecules. White circle represents the critical solution point. Parameters are fixed at $n_{\mathrm{A}}=n_{\mathrm{B}}=10, n_{\mathrm{A}}^{*}=n_{\mathrm{B}}^{*}=1, \lambda_{0}=30.0, C=0.3, C_{1}=-0.5, C_{2}=0.05$, and $\alpha=0.5$.

At intermediate temperature, the two coexistence regions merge, but the $\mathrm{U}$ region and the $\mathrm{U}^{\prime}$ region remain separate. From such a structure of the phase diagram, two-step phase separation is possible; the mixture first separates into two metastable I phases or metastable I and $\mathrm{N}$ phase, and then into stable I and $\mathrm{N}$ phase. For example, when the mixture is quenched to black triangle point in Figure 5, it separates temporarily into metastable I and $\mathrm{N}$ phase (white square) by the driving force based on the $\mathrm{I} / \mathrm{N}$ transition, and eventually into stable I and $\mathrm{N}$ phase (black square) by cooperative driving force due to the I/N transition and the demixing. It is, however, also possible that larger fluctuations in spinodal decomposition lead to direct separation into stable I and $\mathrm{N}$ phase.

At lower temperature, two unstable regions $\mathrm{U}$ and $\mathrm{U}^{\prime}$ also merge, so that the mixture separates directly into stable I and $\mathrm{N}$ phase, or into stable I and $\mathrm{S}_{\mathrm{m}}$ phase by the cooperative driving force.

If we divide the phase diagram into two at the middle and see the left half, it is similar to a theoretical phase diagram of a lyotropic liquid crystal first derived by Flory ${ }^{50}$, and later confirmed by an experiment by Miller et al. ${ }^{51}$ The narrow $\mathrm{I} / \mathrm{N}$ coexisting region extending from the macroscopic phase separation region is called "chimney". In lyotropic liquid crystals, the chimney goes straight up to high temperature, but our results show that in supramolecular liquid crystals there is a limiting temperature of chimney, because the number of mesogenic cores decreases with increasing tempera- ture.

\section{THERMOREVERSIBLE GELATION}

Let us move onto gelling solutions. We first study simple pairwise association of functional groups on polymer chains. Then, we generalize our theory to multiple association. Finally, we study more general mixtures where networks are formed by two species of polymers.

\section{Self-Association of Polymers}

To study self assembly of polymer chains in solvents, we consider a special case of our model binary mixture ${ }^{10-12}$ in which solute molecules A with the molecular weight $n_{\mathrm{A}} \equiv n$, each carrying $f$ identical functional groups, are mixed with low molecular weight $\left(n_{\mathrm{B}}=1\right)$ solvent molecules $B$. Pairwise association only between A groups is assumed $\left(\lambda_{\mathrm{AA}}>0, \lambda_{\mathrm{AB}}=\lambda_{\mathrm{BB}}=0\right)$. In equilibrium state we have solvent $(0,1)$ and $l$-cluster $(l, 0)$, where $l=1,2,3 \ldots$. For simplicity, we contract the double suffices into single ones, and write $l$ for an $l$-cluster, 0 for a solvent. We start from the free energy

$$
\begin{aligned}
\beta \Delta F= & \sum_{l \geq 1} N_{l} \ln \phi_{l}+N_{0} \ln \phi_{0}+\chi \phi(1-\phi) \Omega \\
& +\sum_{l \geq 1} \Delta_{l} N_{l}+\delta(\phi) N^{G}
\end{aligned}
$$

where $N^{\mathrm{G}}$ is the number of A molecules in the macro- 
scopic cluster. Such a macroscopic cluster has infinite molecular weight and can take any geometry; it can be a three dimensional branched network, an infinitely long string, etc.

The multiple equilibrium condition leads to the volume fraction of the clusters to be given by

$$
\phi_{l}=K_{l} \phi_{1}^{l},
$$

written in terms of the volume concentration $\phi_{1}$ of the isolated molecules and the equilibrium constant $K_{l}=$ $\exp \left(l-1-\Delta_{l}\right)$

We now consider the total amount of materials in the sol:

$$
\phi^{\mathrm{S}}(x, y)=y+\sum_{l=1}^{\infty} K_{l} x^{l},
$$

where $x \equiv \phi_{1}$ for the solute and $y \equiv 1-\phi$ for the solvent. To study the infinite summation in this equation, let us define the free energy gain $\delta_{l} \equiv \Delta_{l} / l$ when a single chain participates in a cluster of the size $l$.

Application of the Cauchy-Hadamard's theorem 52 gives the convergence radius $x^{*}$ of the power series in the form

$$
1 / x^{*}=\varlimsup_{l \rightarrow \infty}\left(K_{l}\right)^{1 / l}=e^{1-\delta^{*}},
$$

where the least upper bound of the limit has been indicated by a bar. The quatity $\delta^{*} \equiv \lim _{l \rightarrow \infty} \delta_{l}$ is defined by the limiting value of $\delta_{l}$ as $l \rightarrow \infty$ In this special case of homopolymer association, we have a linear boundary in the unit square on the $(x, y)$ plane which is parallel to the $y$-axis. Within the radius of convergence, the normalization condition $\phi^{S}(x, y)=1$ gives a one to one relationship between $\phi$ and $x$.

Formation of several different spatial structures can be seen from the behavior of $\delta_{l}$. Figure 6 schematically shows the exponent $\delta_{l}+1 / l-1$ of the equilibrium constant $K_{l}^{-1 / l}$ as a function of $l$. This function may either take a minimum at a certain finite $l$ (curve (I) and (III) of Figure 6) or decreases monotonically to a finite value $\delta^{*}-1$ (curve (II)). Let $l_{0}$ be the value of $l$ at which the curve reaches the minimum (including $l_{0}=\infty$ for the monotonic case). The cluster size $l$ for which the volume concentration $\phi_{l}$ becomes largest is given by

$$
\partial \Delta_{l} / \partial l=1+\log x .
$$

Let $l^{*}$ be the solution of this equation.

In the case where $l^{*}$ is finite, the upper bound $x^{*}$, when expressed in terms of the total concentration, is called critical micelle concentration $(\mathrm{cmc})$, since the volume fraction of the clusters with aggregation number $l^{*}$ goes up to a finite fraction at this value of the volume fraction. ${ }^{53}$ Sharpness in their appearance is controlled by the curvature of the function $\delta_{l}+1 / l-1$ around $l^{*}$. At $\mathrm{cmc}$, we have $l_{0}=l^{*}$.

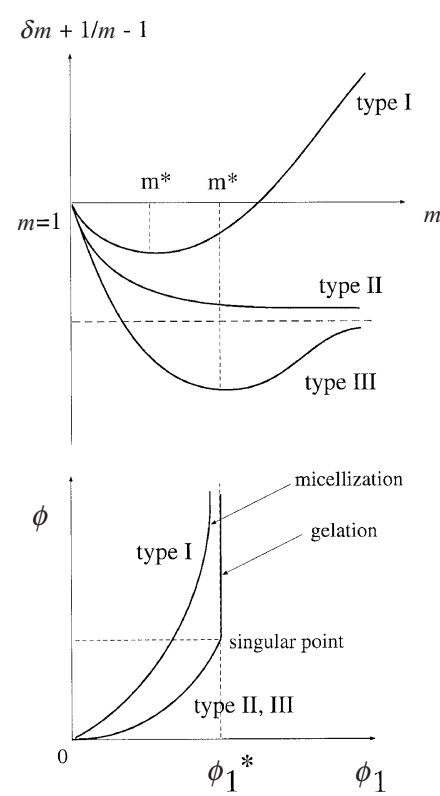

Figure 6. The binding free energy per molecule as functions of the aggregation number. Type I leads to micellization with finite aggregation number. Type II and Type III lead to macroscopic aggregates, such as infinitely long cylindrical micelles, three dimensional networks. In the latter case, the volume fraction $\phi_{1}$ of the molecules that remain unassociated in the solution as a function of the total volume fraction $\phi$ of the molecules shows a singularity at the point where the weight average molecular weight of aggregates becomes infinite.

In the case where $l^{*}$ is infinite, on the contrary, a macroscopic cluster appears as soon as $x$ exceeds the critical value $x^{*} \equiv \exp \left(\delta^{*}-1\right)$.

The macroscopic clusters can be branched networks (gels), ${ }^{4,5}$ or infinitely long polymers ${ }^{128}$ and mice1les. ${ }^{5-57}$ For brevity, we call the former case gelation and the latter case polymerization (including wormlike micellization). The total concentration $\phi^{*}$ obtained from $x^{*}$ gives the concentration at which this transition takes place. It depends on the temperature through $\delta^{*}$. For $\phi$ above $\phi^{*}$, the sum in $\phi^{S}(x, y)$ cannot reach $\phi$. The amount of shortage $\phi-\sum \phi_{l}$ goes into the macroscopic clusters.

\section{Application of Stockmayer's Distribution}

The simplest gelling binary mixture is a polymer solution in which polymers form homopolymer networks in solvents..$^{10,11}$

In order to derive the equilibrium constants, let us introduce a simple model for the internal structure of clusters. For the species A, we consider the identical molecules made up of $n$ statistical monomeric units. Each molecule is carrying $f$ functional groups capable of pairwisely forming bonds. A cluster is assumed to take a tree structure with no internal loops (called "Cayley tree"). This is a crude approximation based on the 
classical molecular-field picture of gelation. ${ }^{8,58,59}$

We split the free energy into three parts:

$$
\Delta_{l}=\Delta_{l}^{\mathrm{comb}}+\Delta_{l}^{\mathrm{conf}}+\Delta_{l}^{\mathrm{bond}}
$$

as before.

To find the combinatorial part, we employ the entropy for combining $l$ identical $f$-functional molecules to form a single Cayley tree. The classical tree statistics gives

$$
\Delta s_{l}^{\mathrm{comb}}=k_{\mathrm{B}} \ln \left[f^{l} \omega_{l}\right],
$$

where

$$
\omega_{l} \equiv \frac{(f l-l) !}{l !(f l-2 l+2) !}
$$

is Stockmayer's combinatorial factor. ${ }^{59}$ The free energy is given by $\Delta_{l}^{\mathrm{comb}}=\Delta s_{l}^{\mathrm{comb}}$.

For the conformational free energy, we employ the lattice theoretical entropy of disorientation ${ }^{6,8} s_{\text {dis }}(n)$ for a polymer with $n$ statistical units, and find

$$
\Delta s_{l}^{\mathrm{conf}}=s_{\mathrm{dis}}(l n)-l s_{\mathrm{dis}}(n)=k_{\mathrm{B}} \ln \left[\left(\frac{\sigma(z-1)^{2}}{z e n}\right)^{l-1} l\right] .
$$

Finally, free energy of bonding is given by

$$
\Delta_{l}^{\text {bond }}=(l-1) \beta \Delta f_{0}
$$

because there are $l-1$ bonds in a tree of $l$ molecules, where $\Delta f_{0}$ is the free energy change for a single bond formation.

Combining all results together, we find

$$
K_{l}=f l \omega_{l}\left(\frac{f \lambda}{n}\right)^{l-1},
$$

where

$$
\lambda(T) \equiv\left[\sigma(z-1)^{2} / z e\right] \exp \left(-\beta \Delta f_{0}\right)
$$

is a temperature dependent constant. We call this constant association constant.

The distribution of clusters eq 27 then gives for the number density

$$
\lambda v_{l}=\omega_{l} x^{l},
$$

where the independent variable $x$ here is defined by $x \equiv$ $f \lambda \phi_{1} / n=f \lambda v_{1}$, which gives the number of functional groups $f \phi_{1} / n$ carried by the isolated polymer chains in the solution multiplied by a temperature shift factor $\lambda(T)$.

From this distribution function, we can obtain average values of physical quantities. First, the total number concentration of the finite clusters is given by

$$
\lambda \sum_{l \geq 1} v_{l}=S_{0}(x)
$$

Their volume fraction is

$$
\frac{\lambda}{n} \sum_{l \geq 1} \phi_{l}=S_{1}(x) .
$$

Therefore, the number average of the cluster size is given by

$$
\overline{l_{n}} \equiv \sum l v_{l} / \sum v_{l}=S_{1}(x) / S_{0}(x)
$$

and the weight average is

$$
\bar{l}_{w} \equiv \sum l^{2} v_{l} / \sum l v_{l}=S_{2}(x) / S_{1}(x) .
$$

These are written in terms of the moments

$$
S_{k}(x) \equiv \sum_{l=1}^{\infty} l^{k} \omega_{l} x^{l} \quad(k=0,1,2 \cdots)
$$

of the Stockmayer distribution.

These moments are most easily expressed by the extent $\alpha$ of reaction defined by the equation

$$
x \equiv \alpha(1-\alpha)^{f-2} .
$$

As a function of $\alpha, x$ takes a maximum value $x^{*}=(f-$ $2)^{f-2} /(f-1)^{f-1}$ at $\alpha=1 /(f-1)$. Therefore, two values of $\alpha$ are decided for a given value of $x$. Let us consider the smaller one for a while. We then have $0 \leq \alpha \leq$ $1 /(f-1)$.

By using $\alpha$ the average cluster sizes are given by

$$
\begin{aligned}
& \bar{l}_{n}=1 /(1-f \alpha / 2) \equiv P_{n}(\alpha) \\
& \bar{l}_{w}=(1+\alpha) /[1-(f-1) \alpha] \equiv P_{w}(\alpha) .
\end{aligned}
$$

The weight average diverges at $\alpha=1 /(f-1)$. This suggests that $\alpha=\alpha^{*} \equiv 1 /(f-1)$ is the gel point. The number average also diverges at $\alpha=2 / f$, but since $2 / f>1 /(f-1)$, we have to study the postgel regime to examine it. In the pregel regime where $\alpha<\alpha^{*}$ holds, the volume fraction $\phi^{\mathrm{S}}$ occupied by the polymer chains belonging to the sol must always equal the total polymer volume fraction $\phi$, since no gel network exists. Thus the total polymer volume fraction $\phi$ and the extent of association $\alpha$ satisfy the relation

$$
\frac{f \lambda}{n} \phi=\frac{\alpha}{(1-\alpha)^{2}} .
$$

We can solve this equation for $\alpha$. The result can be expressed as

$$
\alpha=\frac{1}{2 c}\{1+2 c-\sqrt{1+4 c}\}
$$

in terms of the number concentration

$$
c \equiv \frac{f \lambda(T)}{n} \phi
$$

of the functional groups (multiplied by the association constant). We can then express all physical quantities directly in terms of $c$. 
Let us next find the sol/gel transition point. The free energy change $\delta_{l}$ per molecule is a steadily decreasing function of $l$, approarching the limiting value $\delta^{*}=1-$ $(f-1) \log (f-1)+(f-2) \log (f-2)-\log \lambda(T)$. This gives for the convergence radius of the series eq 60

$$
\phi_{1}^{*}=\exp \left(\delta_{\infty}-1\right)
$$

or, equivalently,

$$
x=x^{*}=(f-2)^{f-2} /(f-1)^{f-1}
$$

in terms of $x$, and

$$
\alpha=\alpha^{*}=1 /(f-1)
$$

in terms of the extent of association as was expected above from the divergence of $\bar{l}_{w}$. The volume fraction of polymers at the gel point is then given by

$$
\frac{\lambda}{n} \phi^{*}=\frac{f-1}{f(f-2)^{2}}
$$

This gives sol/gel transition line on the temperatureconcentration plane.

Postgel Regime - Stockmayer's Treatment vs. Flory's Treatment -

In the postgel regime where $\phi>\phi^{*}$ or $\alpha>\alpha^{*}$, we have additional balance condition between the chamical potential of a polymer chain in the sol part and the one in the gel part. ${ }^{60}$ This equilibrium condition $\Delta \mu_{1}=\Delta \mu^{\mathrm{G}}$ gives $\ln \phi_{1}=\delta(\phi)-1$. The volume fraction of the unreacted chains is related to the binding free energy of a chain onto the gel network.

Since the conversion in the sol can be different from that in the gel, let us write the former as $\alpha^{\prime}$ and the latter as $\alpha^{\prime \prime}$. The average conversion $\alpha$ of the solution as a whole is then given by

$$
\alpha=\alpha^{\prime} w_{\mathrm{S}}+\alpha^{\prime \prime} w_{\mathrm{G}} .
$$

The volume fraction $\phi^{\mathrm{S}}$ of polymers belonging to the sol is consequently given by

$$
\lambda \phi^{\mathrm{S}} / n=S_{1}\left(\alpha^{\prime}\right)
$$

in the postgel regime, so that it is different from the total $\phi$ given in terms of $\alpha$. The sol fraction $w_{\mathrm{S}}$ here is defined by

$$
w_{\mathrm{S}} \equiv \phi^{\mathrm{S}} / \phi=S_{1}\left(\alpha^{\prime}\right) / S_{1}(\alpha),
$$

and hence the gel fraction is given by

$$
w_{\mathrm{G}}=1-S_{1}\left(\alpha^{\prime}\right) / S_{1}(\alpha) \text {. }
$$

The number $v^{\mathrm{S}}$ in the chemical potentials must also be replaced by

$$
v^{\mathrm{S}}=S_{0}\left(\alpha^{\prime}\right) / \lambda+1-\phi
$$

since it gives the number of objects that have translational degree of freedom.

\section{Flory's Treatment}

To find the extent of reaction in the sol and in the gel, Flory considered ${ }^{8}$ that the smaller root $\alpha^{\prime}$ of the equation

$$
x \equiv \alpha(1-\alpha)^{f-2}
$$

for a given physically acceptable $x$ corresponds to the extent of reaction in the sol, and the larger one $\alpha$ gives the average extent of reaction for all functional groups in the system. The extent of association $\alpha^{\prime \prime}$ in the gel is given by the eq 52 .

The relation

$$
\alpha^{\prime}\left(1-\alpha^{\prime}\right)^{f-2}=\alpha(1-\alpha)^{f-2}
$$

holds by definition. The volume fraction $\phi^{\mathrm{S}}$ of polymers in the sol is then given by

$$
\frac{\lambda}{n} \phi^{\mathrm{S}}=\frac{\alpha^{\prime}}{f\left(1-\alpha^{\prime}\right)^{2}} .
$$

On the contrary, the total polymer volume fraction $\phi$ and the average extent of association $\alpha$ must be given by eq 47 .

\section{Stockmayer's Treatment}

However, Stockmayer ${ }^{59}$ later remarked that Flory's result in the postgel regime is inconsistent with the tree assumption, since the treatment permits cycle formation in the gel network. To remove this inconsistency, he proposed another treatment of the postgel regime. He introduced a different assumption that the extent of reaction of functional groups in the finite clusters remains at $1 /(f-1)$, its value at the gel point, i.e., $\alpha^{\prime}=\alpha^{*}$. He also proposed that in the postgel regime the extent of association in the gel network takes the value $\alpha^{\prime \prime}=2 / f$ appropriate to an infinite tree without cyclic structures. The weight fraction $w_{\mathrm{G}}$ of the gel then takes the form

$$
w_{\mathrm{G}}=\frac{(f-1) \alpha-1}{1-2 / f},
$$

where $\alpha\left(>\alpha^{*}\right)$ is the extent of reaction with respect to the all functional groups. The volume fraction of the sol $\phi^{\mathrm{S}}$ remains constant at $\phi^{\mathrm{S}}=\phi^{*}$. From a physical standpoint, Flory's model is closer to reality, since intramolecular connections are an essential feature of the network structure.

Figure 7 compares phase diagrams calculated by the two different treatments of the postgel regime. ${ }^{60}$ The binodals and spinodals appear in different positions. For the same association constant, Stockmayer's treatment gives a tricritical point (TCP) ${ }^{61,62}$ at the crossing of sol/gel transition line and binodal (spinodal), while Flory's treatment gives a critical endpoint $(\mathrm{CEP})^{62}$ at 


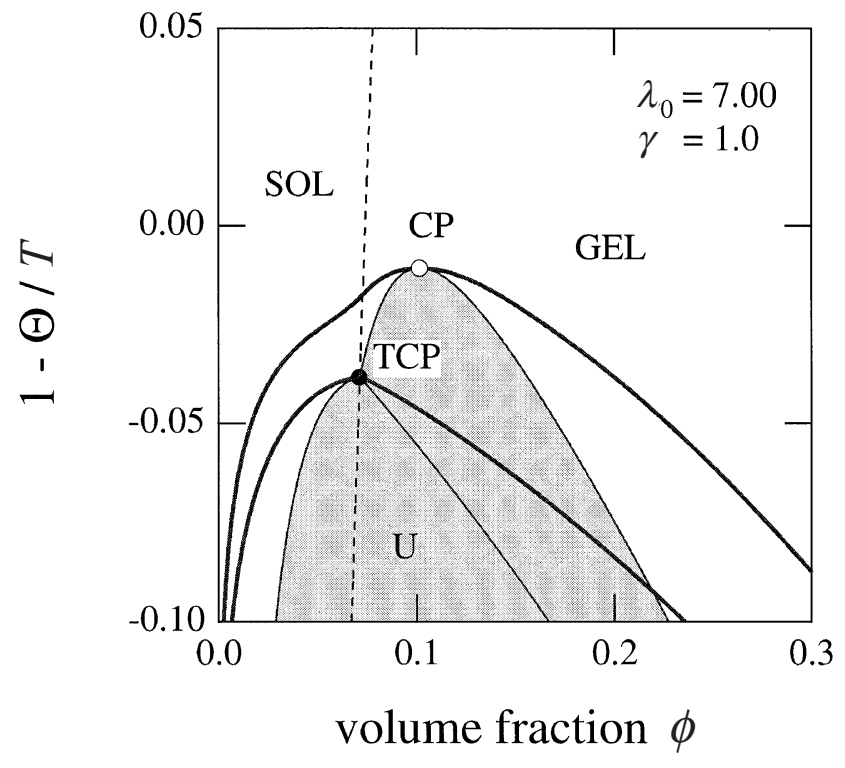

Figure 7. Comparison of the theoretical phase diagrams calculated by Flory's treatment and Stockmayer's treatment of the postgel regime for the same association constant. Flory's treatment allows cycle formation within the gel network, so that phase separation between dilute gel (with only a few cycles) and concentrated gel (with many cycles) with a critical solution point (white circle indicated by $\mathrm{CP}$ ) is possible in the postgel regime.

the shoulder of the binodal and a critical point (CP) in the postgel regime. Flory's treatment allows cycle formation within the gel network, so that phase separation between dilute gel (with only a few cycles) and concentrated gel (with many cycles) in the postgel regime.

In Figure 8, we show a comparison between experimental phase diagram of atactic polystyrene (atPS) solution in carbon disulfide $\left(\mathrm{CS}_{2}\right)^{63-65}$ and its theoretical calculation. This solution shows TCP type phase diagram, but CEP types were also reported for at-PS in different solvents. ${ }^{64}$ The molecular mechanism of cross-linking has been the subject of a great deal of works, ${ }^{4,64,66,67}$ but there still remains a divergence in opinions. One series of studies postulate ${ }^{66}$ the existence of short crystallizable stereoregular segment sequences on polymer chains, even if they are atactic, that are responsible for the formation of microcrystalline junctions. Another studies ${ }^{4,67}$ propose that cross-linking takes place by specific interaction such as formation of stoichiometric compounds involving solvent molecules. If such complex formation is the mechanism of cross-linking, the gelation temperature should not show a steadily increasing function of the polymer concentration, but should show a maximum at a certain concentration. ${ }^{67}$ Existence of specific interaction was also suggested by light scattering study of at-PS dilute solution in toluene- $\mathrm{CS}_{2}$ mixture. ${ }^{68}$
(atactic)Polystyrene/CS

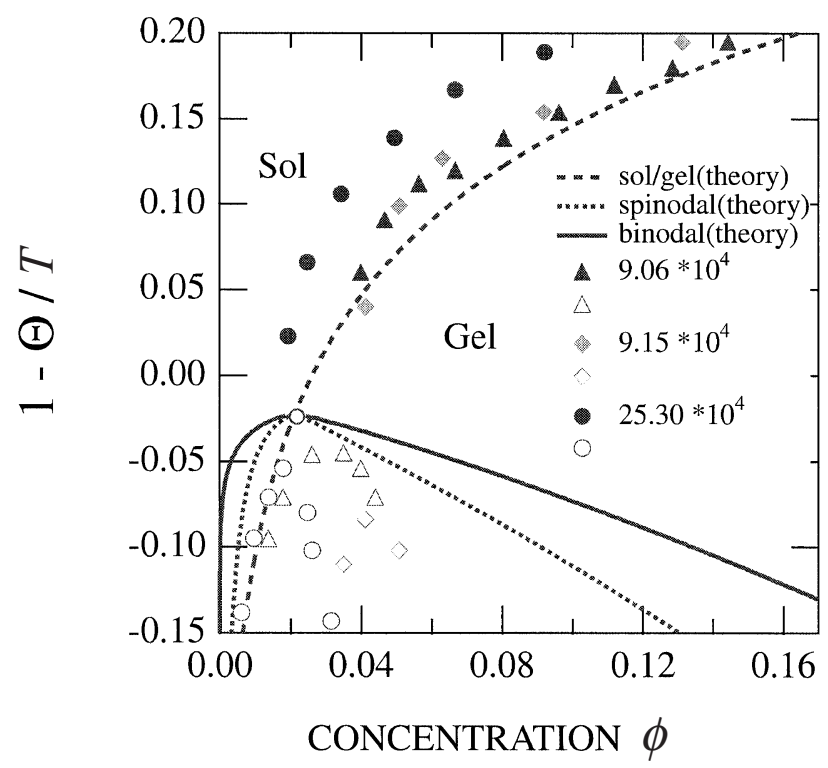

Figure 8. Phase diagram of atactic polystyrene in carbon disulfide. Thermoreversible gelation coexist with phase separation. Theoretical sol/gel transition line (broken line), binodal (solid line) and spinodal (dotted line) are drawn. Temperature is measured by the reduced temperature $\tau \equiv 1-\Theta / T$ with the theta tempearature $\Theta=-70^{\circ} \mathrm{C}$. Experimental data of the gel points (black symbols) and cloud points (white symbols) are shown for three different molecular weights. Theoretical calculation is fitted to the data for $M=9.06 \times 10^{4}$.

\section{THERMOREVERSIBLE GELATION WITH MULTIPLE JUNCTIONS}

Most thermoreversible gels observed so far are crosslinked by the formation of network junctions involving polymer segments belonging to several distinct chains(multiple junctions). For instance, gelation by micro-crystallization of the chain segments, by ionic aggregation, and by hydrophobic association of special groups attached on the polymer chains, all belong to this important category. ${ }^{4,5}$

Among these, associating polymers are very important, because they form ultraweak networks in water. The associating polymers are water-soluble polymers carrying hydrophobic groups on the backbone or on the chain side. ${ }^{13,69}$

Typical model APs that have recently been the focus of study are water-soluble polymers partially modified by hydrophobic groups. One series of APs are based on poly(ethylene oxide) chains (referred to as PEO), being modified by short alkyl chains, ${ }^{70-74,125}$ propyrene oxide (or butylene oxide) chains ${ }^{77}$ and fluorocarbon chains. ${ }^{78-81}$ Hydrophobes are either period- 
ically or randomly attached on a polymer chain. The simplest one is a telechelic polymer carrying two hydrophobes at the chain ends. Another series of APs are based on cellulose derivatives. Some examples are ethyl hydroxyethyl cellulose (EHEC) ${ }^{82-84}$ and hydroxypropyl methyl cellulose (HPMC). ${ }^{85,86}$ Polyelectrolytes partially modified by hydrophobic groups have also been intensely studied. ${ }^{87-91}$

Associative groups form aggregates, or micelles, through hydrogen bonds, ionic attraction, hydrophobic interaction, etc. Polymers with associative interactions exhibit a variety of condensed phases, typical examples of which are microscopically ordered phases, gels, and liquid crystals. ${ }^{2-5}$ All of these phases have their counterparts formed by covalently bonded polymers, but, since association is thermally controllable, associating phases provide a new pathway to modelling statistical clusters, block copolymers, and reversible networks. APs also exhibit characteristic rheological properties such as shear thickening ${ }^{70,76,125}$ at relatively low shear rate, so that they imply vast industrial applications as rheology control agents.

In this section, we extend our theory of networkforming polymer solutions from pairwise association to more general multiple association.

\section{Multiple Association}

As a model solution, we consider a mixture of associative molecules in a solvent. Molecules are distinguished by the number $f$ of the associative (functional) groups they bear, each associative group being capable of taking part in the junctions with variable multiplicity which may bind together any number $k$ of such groups. 13,92-94 We include $k=1$ representing unassociated groups. In what follows, we allow junctions of all multiplicities to coexist, in proportions determined by the thermodynamic equilibrium conditions. Let $n_{f}$ be the number of the statistical segments on an $f$-functional molecule, and let $N_{f}$ be the number of $f$-functional primary molecules in the solution. The weight fraction $w_{\mathrm{f}}$ of the associative groups carried by the molecules with specified $f$ relative to the total number of associative groups is then given by

$$
w_{f}=f N_{f} / \sum f N_{f} .
$$

The number and weight average functionality of the primary molecules are then defined by

$$
f_{\mathrm{n}} \equiv\left(\sum w_{f} / f\right)^{-1}
$$

and

$$
f_{\mathrm{w}} \equiv \sum f w_{f}
$$

In thermal equilibrium, the solution has a distribution of clusters with a population distribution fixed by the equilibrium conditions. Following the notation used by Fukui and Yamabe, ${ }^{92}$ we define a cluster of type $(\mathbf{j}, \mathbf{l})$ to consist of $j_{k}$ junctions of multiplicity $k$ $(k=1,2,3, \ldots)$ and $l_{f}$ molecules of functionality $f$ $(f=1,2,3, \ldots)$. The bold letters $\mathbf{j} \equiv\left\{j_{1}, j_{2}, j_{3}, \ldots\right\}$ and $\mathbf{I} \equiv\left\{l_{1}, l_{2}, l_{3}, \ldots\right\}$ denote the sets of indices. An isolated molecule of functionality $f$, for instance, is indicated by $\mathbf{j}_{0 f} \equiv\{f, 0,0, \ldots\}$, and $\mathbf{l}_{0 f} \equiv\{0, \ldots, 1,0, \ldots\}$.

The multiplicity of junctions is in principle determined by the equilibrium requirement for a given associative interaction. In the case of hydrophobic interaction, the chain length of a hydrophobe, the strength of water-hydrophobe interaction, the geometric form of an aggregate, and other factors determine the association constant $\lambda(T)$ and the multiplicity of junctions. In the present theoretical study, we avoid complexity in finding the precise distribution of the multiplicity, but instead, we introduce a model junction ${ }^{13,93,94}$ in which multiplicities lying in a certain range covering from $k=s_{\min }$ to $s_{\max }$ are equally allowed. We thus have

$$
k=1 \quad(\text { free }), \quad k=s_{\min }, s_{\min }+1, \ldots, s_{\max }
$$

(associated).

When only a single value is allowed, i.e., $s_{\min }=s_{\max } \equiv$ $s$, we call the model fixed multiplicity model. Thus, for $s=2$, fixed multiplicity model reduces to pairwise association. Such assumption of mini-max junction can be to some extent justified in the molecular simulation of AP described below by using a simple attractive potential among associative groups. ${ }^{13,94}$

Let $N(\mathbf{j} ; \mathbf{l})$ be the number of $(\mathbf{j} ; \mathbf{l})$-clusters in the system. Then their number density is given by $v(\mathbf{j} ; \mathbf{l})=$ $N(\mathbf{j} ; \mathbf{l}) / \Omega$, and their volume fraction is given by

$$
\phi(\mathbf{j} ; \mathbf{l})=\left(\sum_{f \geq 1} n_{f} l_{f}\right) v(\mathbf{j} ; \mathbf{l}),
$$

where $\Omega$ is the total number of lattice cells in the system. The total volume fraction of the polymer component is thus given by $\phi=\sum_{\mathbf{j}, \mathbf{l}} \phi(\mathbf{j} ; \mathbf{l})$.

\section{Theoretical Criterion of the Gel Point}

As in the preceeding sections we start from the standard reference state (polymers and solvent molecules being separated in hypothetical crystalline states). The free energy change on passing from this reference state to the final solution, at equilibrium with respect to cluster formation, is given by the expression 
$\frac{\beta \Delta F}{\Omega}=v_{0} \ln \phi_{0}+\sum_{\mathbf{j}, \mathbf{l}} v(\mathbf{j} ; \mathbf{l})[\Delta(\mathbf{j} ; \mathbf{l})+\ln \phi(\mathbf{j} ; \mathbf{l})]+\chi \phi_{0} \phi$

in the pregel regime. ${ }^{93}$ The free energy change $\Delta(\mathbf{j} ; \mathbf{l})$ accompanying the formation of a $(\mathbf{j} ; \mathbf{l})$-cluster in a hypothetical undiluted amorphous state from the separate primary molecules in their standard states is defined by

$$
\Delta(\mathbf{j} ; \mathbf{l}) \equiv \beta\left\{\mu^{\circ}(\mathbf{j} ; \mathbf{l})-\sum_{f} l_{f} \mu^{\circ}\left(\mathbf{j}_{0 f} ; \mathbf{l}_{0 f}\right)\right\} .
$$

In the postgel regime where a cluster grows to a macroscopic network, the free energy has an additional contribution from the gel part. ${ }^{12,93}$

Now, the chemical equilibrium conditions are given by

$$
\Delta \mu(\mathbf{j} ; \mathbf{l})=\sum l_{f} \Delta \mu\left(\mathbf{j}_{0 f} ; \mathbf{l}_{0 f}\right) .
$$

Now the free energy $\Delta(\mathbf{j} ; \mathbf{l})$ consists of three parts: combinatorial, conformational, and bonding ones. To use the multiple tree statistics ${ }^{92}$ for the combinatorial entropy of cluster formation, we assume that all clusters take tree form as in the classical literature. ${ }^{58,59}$

To summerize the result, we introduce the extent $\alpha$ of association, or conversion, that is defined by the probability for a randomly chosen associative group to be associated. Let $p_{k}$ be the probability for a randomly chosen group to be in the junction of multiplicity $k$. The extent $\alpha$ is then expressed as

$$
\alpha \equiv \sum_{k \geq 2} p_{k} .
$$

Then, $p_{1} \equiv 1-\alpha$ is the probability for a hydrophobe to remain unassociated. This is equivalent to the normalization condition $\sum p_{k}=1$. This condition indicates that the total concentration $\psi$ of the hydrophobes should satisfy the relation

$$
\lambda(T) \psi=z u(z),
$$

where the function $u(z)$ to be used to characterize junctions is defined by

$$
u(z) \equiv \sum_{k=1}^{\infty} \gamma_{k} z^{k-1} .
$$

The coefficient $\gamma_{k}$ comes from the surface free energy of an aggregate with multiplicity $k$. The parameter $z$ that appeared in the above relations is defined by $z \equiv \lambda(T) \psi p_{1}=\lambda(T) \psi(1-\alpha)$, and gives the (reduced) concentration of the hydrophobes that remain unassociated in the solution.

The next step is to calculate the weight-average molecular weight of the clusters. From its divergence, we find the sol/gel transition point. It is most generally given by ${ }^{92,93}$

$$
\left(f_{\mathrm{w}}-1\right)\left(\mu_{\mathrm{w}}-1\right)=1,
$$

where $f_{\mathrm{w}}$ defined by eq 63 is the weight average functionality of the primary chains, and $\mu_{\mathrm{w}} \equiv \sum_{k \geq 1} k p_{k}$ the average multiplicity of the junctions. For monodisperse functionality, this equation reduces to

$$
(f-1) z u^{\prime}(z) / u(z)=1 .
$$

For a specific model of mini-max junction eq 64 , we have

$$
u(z)=1+\sum_{k=s_{\min }}^{s_{\max }} z^{k-1}=1+\left(z^{s_{\min }-1}-z^{s_{\max }}\right) /(1-z)
$$

by neglecting possible contributions from micellar surfaces and setting all $\gamma_{k}=1$ for $s_{\min } \leq k \leq s_{\max }$.

For example, the above normalization relation for the fixed multiplicity model of monodisperse polymers $(f$ and $n$ definite) is given by

$$
\lambda(T) \phi / n=\alpha^{1 / s^{\prime}} / f(1-\alpha)^{s / s^{\prime}},
$$

which connects the extent $\alpha$ of association to the (scaled) polymer concentration. From here on, abbreviations $f^{\prime} \equiv f-1$ and $s^{\prime} \equiv s-1$ are used.

The gel point condition eq 73 gives $f^{\prime} s^{\prime} \alpha=1$ and hence $\alpha=\alpha^{*} \equiv 1 / f^{\prime} s^{\prime}$ leading to the critical concentration

$$
\lambda(T) \phi^{*} / n=f^{\prime} s^{\prime} / f\left(f^{\prime} s^{\prime}-1\right)^{s / s^{\prime}},
$$

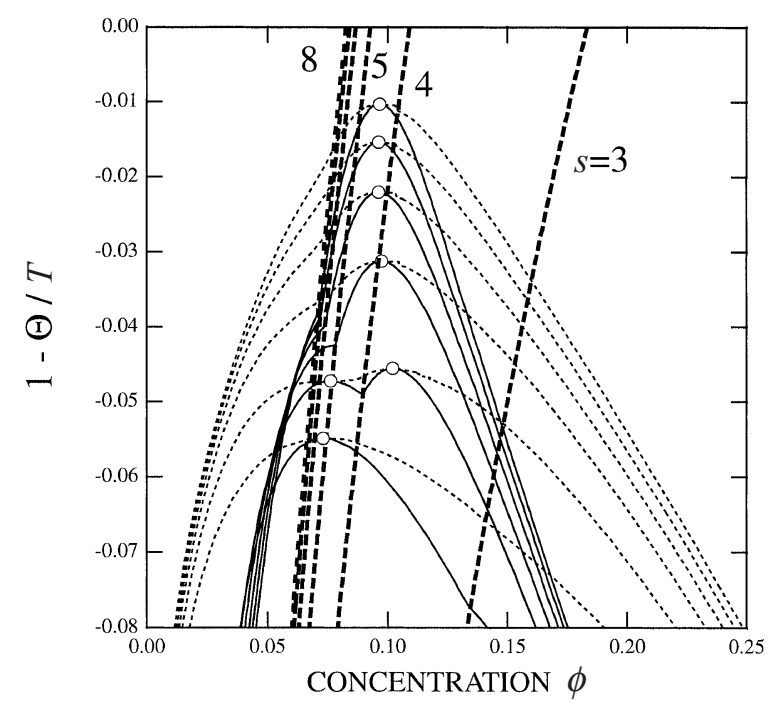

Figure 9. Sol/gel transition lines (thick broken lines), binodals (thin broken lines) and spinodal lines (solid lines) of bifunctional ( $f=2$ ) polymers with $n=100, \lambda_{0}=10.0$ for association with fixed multiplicity $\left(s_{\min }=s_{\max } \equiv s\right.$ ). Multiplicity $s$ is changed from 3 to 8 . The transition line shifts to high temperature and low concentration region with the multiplicity. Gelation is easier for larger multiplicity. 
where $\phi^{*}$ is the volume fraction of the polymer at gelation. As the multiplicity is changed, with other parameters kept fixed, gelation concentration changes and the sol/gel line shifts on the temperature-concentration plane (Figure 9).

Phase equilibria and thermodynamic stability can be studied by using chemical potentials of the polymer and of the solvent. These are derived above by differentiating the free energy. Binodal curves and spinodal curves can then be drawn on the temperature and concentration plane. In such AP solutions, gelation and phase separation generally compete with each other, and as a result phase diagrams with higher order critical points such as tricritical point ${ }^{61,62}$ and critical endpoint ${ }^{62}$ are derived. ${ }^{93}$

\section{STRUCTURE OF THE NETWORKS WITH MULTIPLE JUNCTIONS}

On passing the gel point, networks appear and coexist with finite clusters. The structure of a network can be studied from two different viewpoints: local viewpoint and global one. The local structure of a network focuses on the structure of each network junction, including its multiplicity, sequence length, degree of chain packing, etc., while the global structure treats topological connectivity of the network as a whole, paying special attention to the cycle rank (number of independent loops), number of elastically effective chains, number of dangling ends, average path number of junctions, etc. Studies from such different viewpoints are complementary to each other, and both are necessary.

\section{Local Structure of Networks - Augmented Eldridge- Ferry Method -}

When an associative group on a chain involves $\zeta$ sequential repeat units, we can write the standard free energy change as in eq 2. By taking the logarithm of the gelation concentration eq 76, we find an important relation

$$
\ln \phi^{*}=\zeta \frac{\Delta h}{k_{\mathrm{B}} T}+\ln \left[\frac{f^{\prime} s^{\prime} n}{f\left(f^{\prime} s^{\prime}-1\right)^{s / s^{\prime}}}\right]-\zeta \frac{\Delta s}{k_{\mathrm{B}}} .
$$

We can find multiplicity $s$ and sequence length $\zeta$ by comparing this relation with the experimental sol/gel transition concentration. ${ }^{95}$ For the hydrophobes on associating polymers, the enthalpy $\Delta h$ of a cross-link is found because $\zeta$ is known. For the micro-crystalline junction formed by homopolymers, each $\zeta$ sequence of repeat units along a chain surves as a functional group for cross-linking. In such a case, a polymer chain is regarded as carrying $f=n / \zeta$ functional groups. Since

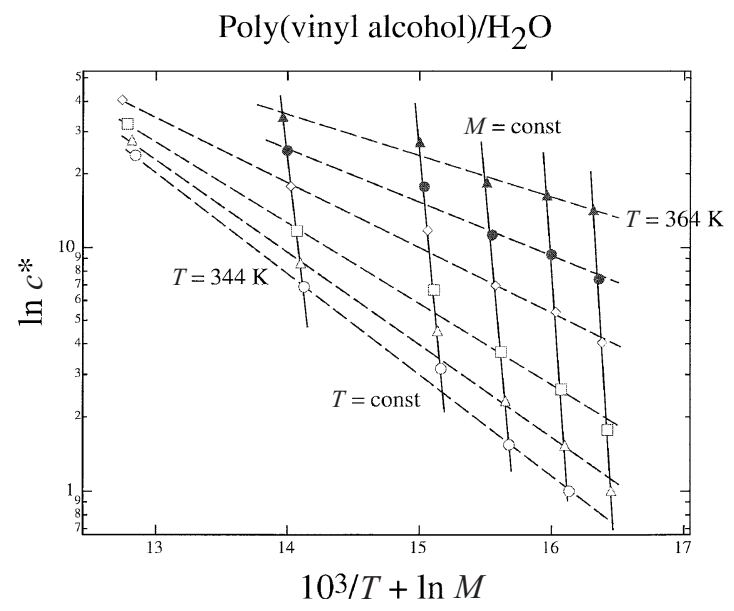

Figure 10. Augmented Eldridge-Ferry plot applied to the gel melting concentration of poly(vinyl alcohol)/water. Dotted lines show the gel melting concentration at constant temperature, while thin straight lines show those at constant molecular weight. (৫) $91^{\circ} \mathrm{C} ;(\bullet) 87^{\circ} \mathrm{C} ;(\diamond) 83^{\circ} \mathrm{C} ;(\square) 78^{\circ} \mathrm{C} ;(\triangle) 74^{\circ} \mathrm{C} ;(\bigcirc) 71^{\circ} \mathrm{C}$.

we have large $n$, and hence large $f$, we can neglect 1 compared to $n$ or $f$, and are led to an equation

$$
\ln c^{*}=\zeta \frac{\Delta h}{k_{\mathrm{B}} T}-\frac{1}{s-1} \ln M+\text { constant }
$$

for micro-crystalline gels, where weight concentration $c^{*}$ has been substituted for the volume fraction. This equation enables us to find $\zeta$ and $s$ independently. For the special case of pairwise association $s=2$, this equation reduces to the conventional Eldridge-Ferry equation. $^{96}$

Let us plot $\ln c^{*}$ against $10^{3} / T+\ln M$. Then the slope $-B$ of the line at constant $T$ gives $-1 /(s-1)$, while the slope $-A$ of the line at constant $M$ gives

$$
\zeta=\frac{10^{3} k_{\mathrm{B}}}{|\Delta h|} A=\frac{10^{3} R}{\left|(\Delta h)_{\mathrm{mol}}\right|} A,
$$

where $(\Delta h)_{\mathrm{mol}}$ is the enthalpy of bonding per mole of the repeat units, and $R$ the gas constant. We have applied this method to experimental data on the gel melting curves of several thermoreversible gels. ${ }^{97}$

As an example of such analysis, we show in Figure 10 the result for the gelation of poly(vinyl alcohol)(PVA) in water. ${ }^{97}$ PVA is known to be a typical crystalline polymer, but it also gels in aqueous solution under large supercooling. There are several pieces of experimental evidence that the crosslinks are formed by partial crystallization of the polymer segments in which syndiotactic sequence dominates, while subchains connecting the junctions consist mainly of atactic non-crystalline sequences on PVA chains. The micro-crystals at the junctions are supposed to be stabilized by hydrogen bonds between the hydroxy groups. We plot the gel melting temperature found from differential scanning calorimetry 
(DSC) and visco-elastic measurements for PVA with different molecular weights covering the range from $2 \times 10^{4}$ to $8 \times 10^{5}$ in various concentrations. ${ }^{98}$ The gel melting temperature $T_{\mathrm{m}}$ is estimated from the temperature at which the DSC heating curve shows an endotherm peak. The slope of the solid lines with constant molecular weight gives $-A=13.43$ almost independently of their molecular weights. Hence we find $\zeta=26.7 \mathrm{kcal} \mathrm{mol}^{-1} / \mid(\Delta h)_{\mathrm{mol}}$. If we use the heat of fusion $(\Delta h)_{\mathrm{mol}}=1.64 \mathrm{kcal} \mathrm{mol}^{-1}$ in the bulk crystal, we find $\zeta=16.3$. On the other hand, the slope of the dotted lines with constant temperature depends on the temperature. At the highest temperature $T=91{ }^{\circ} \mathrm{C}$ in the measurement, it is -0.38 , while it gives a larger value -0.9 at $T=71{ }^{\circ} \mathrm{C}$. The multiplicity is estimated to decrease from 3.6 for high-temperature melting to 2.1 for low-temperature melting, suggesting a very thin junction structure. From thermodynamic stability of the junctions it is natural that a gel which melts at lower temperature has thinner junctions.

\section{Global Structure of Networks - Elastically Effective Chains -}

To study visco-elastic properties of networks, we next find the number $v_{\text {eff }}$ of elastically effective chains. ${ }^{8,99}$ The elastically effective chains are those chains that transmit stress when the network is deformed by external force. They are related to the topological structure of the network. Let us first specify the type of junctions from their connection paths to the network matrix. ${ }^{95}$ A junction of multiplicity $k$ that is connected to the network matrix through $i$ paths is referred to as an $(i, k)$-junction. Let $\mu_{i, k}$ be the number of junctions in the network specified by the type $(i, k)$ for $0 \leq i \leq 2 k$ and for $k=1,2,3,4, \cdots$. The total number of junctions with multiplicity $k$ is given by

$$
\mu_{k}=\sum_{i=0}^{2 k} \mu_{i, k} .
$$

To find the number of elastically effective chains, we next employ the criterion of Scanlan ${ }^{100}$ and Case. ${ }^{101}$ It assumes that only subchains connected at both ends to junctions carrying at least three paths to the gel are elastically effective. We thus have $i, i^{\prime} \geq 3$ for an effective chain. A junction with one path $(i=1)$ to the gel unites a group of subchains dangling from the network matrix whose conformations are not affected by an applied stress. A junction with two paths $(i=2)$ to the gel merely extends the length of an effective subchain. We may call a junction with $i \geq 3$ an elastically effective junction. An effective chain is defined as a chain connecting two effective junctions at both its ends. We thus find

$$
\mu_{\mathrm{eff}}=\sum_{k=2}^{\infty} \sum_{i=3}^{2 k} \mu_{i, k}
$$

for the number of elastically effective junctions, and

$$
\nu_{\mathrm{eff}}=\frac{1}{2} \sum_{k=2}^{\infty} \sum_{i=3}^{2 k} i \mu_{i, k}
$$

for the number of elastically effective chains.

A dangling end may consist either of a single subchain or of a group of subchains connected by several branch points. The structure of a dangling end can be described by the number of subchains and branch points it contains. By definition, the number of dangling ends is given by

$$
v_{\text {end }}=\sum_{k=2}^{\infty} \sum_{i=2}^{2 k}(2 k-i) \mu_{i, k} .
$$

The summation is taken over junctions with $i \geq 2$ because a junction with only one path to the gel is just a branch point on an already counted dangling end.

These topological relations hold for arbitrary networks. Their advantage lies in the fact that, by combinatorial counting, we can actually find $\mu_{i, k}$ as a function of the degree $\alpha$ of association. ${ }^{102}$ In our study, the degree $\alpha$ is found as a function of the temperature and concentration through the relation eq 70 , so that all topological numbers described above can be calculated as functions of the temperature and concentration. ${ }^{95}$ These curves can be compared with the experimental data on the high frequency dynamic modulus measured by Annable et al..$^{70}$ Their experimental data for HEUR C16/35K (PEO end-capped with $\mathrm{C}_{16} \mathrm{H}_{33}$, molecular weight 35000 ) are compared with our theoretical calculation ${ }^{95}$ for $f=2$ in Figure 11. We have chosen as $c^{*}=1.0 \%$ for the weight concentration at gelation. With this gel concentration, the scaling power at the critical region gives $t=1.6$, close to the percolation value. ${ }^{103}$ But since this power depends sensitively on the way we choose $c^{*}$, more detailed experimental examination in the critical region is eagerly required. In fitting the data, we have horizontally shifted the experimental data because of the temperature pre-factor $\lambda(T)$ and also because of the difference in the unit of the polymer concentration. Although fitting by a single theoretical curve is impossible due to the existence of polydispersity in the multiplicity, our theory produces correct behavior over a wide range of the concentration with multiplicity ranging from 6 to 8 . It turns out that about $60 \%$ of chains are elastically effective at the limit of high concentration. 


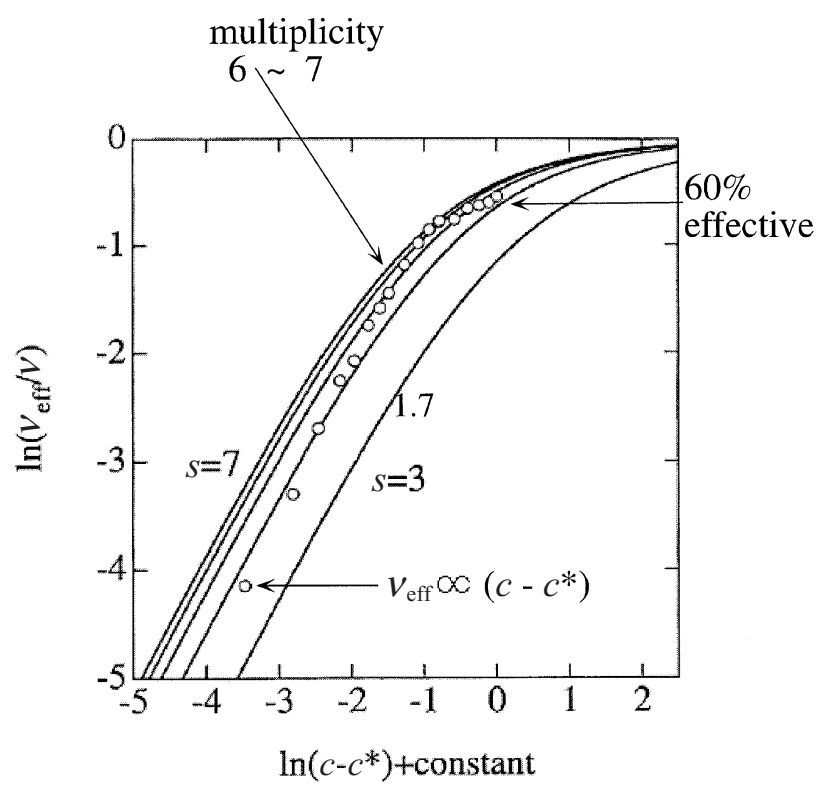

Figure 11. The number of elastically effective chains as a function of the polymer concentration. Experimental data of the HEUR $16 \mathrm{C} / 35 \mathrm{~K}$ are compared with theoretical calculation. In the theoretical calculation the junction multiplicity is changed from curve to curve.

\section{APPLICATION TO COMPLEX THERMOREVERSIBLE GELATION}

The general theory of network-forming polymer solutions and mixtures described above can be refined and augmented to suit for the application to specific problems. In what follows, we summarize some important results obtained so far on complex thermoreversible gels.

\section{Gelation of Hydrated Polymers}

Natural polymers often gel on heating. Typical example which has long been under intensive scrutiny is methyl cellulose with nearly full degree of molar substitution by methoxyl groups. ${ }^{4,85}$ Substitution by large side-chain groups prevents polymers from crystallization. In an aqueous solution at low temperatures, polymer chains are covered with water molecules attached by hydrogen bond (hydration) so that direct association between polymer segments is prevented. As the temperature is raised, chains gradually lose attached waters, and polymer-polymer association, which is initiated by hydrophobic interaction, being stabilized by direct hydrogen bonds, begins to take place. As dehydration proceeds, the number of direct interchain association increases and eventually reaches a critical value for the formation of an infinite network of polymers. It is reversible in the sense that the gel liquefies to the original constituency on cooling. ${ }^{85}$ Because the hydropho- bic segments on polymer chains are partly exposed to water in the gel regime, solution tends to separate into two macroscopic phases. Thus gelation and phase separation compete as the temperature goes up, and the solution reveals interesting multi-critical phase behavior. A typical phase diagram showing co-occurrence of gelation and phase separation, though very crude, was reported in the literature. ${ }^{85,104}$

Quite recently, formation of intra- and interchain hydrogen bonds of cellulose derivatives (6-Omethylcellulose and 2,3-di-O-methylcellulose) was directly observed ${ }^{105}$ by Fourier transform infrared spectroscopy. The study revealed that primary hydroxyl groups at the C- 6 position of cellulose have strong tendency to form interchain hydrogen bonds, while the secondary hydroxyl groups at C-2 and C-3 positions show no evidence of interchain coupling. Thus experimental study of molecular mechanism for the formation of network junctions is presently making a considerable progress.

On the basis of the above molecular picture, we choose $n_{\mathrm{A}}=n$ and an arbitrary $f$ for the polymer chain, and $n_{\mathrm{B}}=1, f=1$ for the solvent molecule. We allow hydrogen bond of the type A.A and A.B, but neglect $\mathrm{B} \cdot \mathrm{B}$ bond assuming that hydrogen bonds among water molecules are weak compared to polymer-water bonds. The association between A groups on the polymer chains must be multiple association if they are hydrophobic groups, but here we assume it pairwise for simplicity. We are now trying to remove this restriction and generalize our theory to multiple cross-linking.

By the tree assumption, $(l, m)$-cluster has $l-1$ crosslinks connecting $l$ constituent polymer chains and $f l-$ $2 l+2$ unreacted functional groups, $m$ of which are hydrated by solvent molecules. We employ Stockmayer's combinatorial factor (33) for the connection of the $f$ functional polymers into a tree. For each connection of the polymers, $m$ attaching sites must be chosen out of $f l-2 l+2$ open sites for hydration. This selection gives a binomial factor $f l-2 l+2 C_{m}$. The total combinatorial free energy change then takes the form

$$
\Delta_{l, m}^{\mathrm{comb}}=-\ln \left[f l-2 l+2 C_{m} f^{l} \omega_{l}\right] .
$$

Upon substitution of this result into our general equations, we find that the number distribution of clusters is given by

$$
v_{l, m}=\frac{1}{\lambda(T)} f l-2 l+2 C_{m} \omega_{l} x^{l} y^{m}+\frac{1}{\mu(T)} y \delta_{l, 0} \delta_{m, 1},
$$

where $\delta$ is the Kronecker's delta. We have introduced two parameters

$$
x \equiv f \lambda(T) \phi_{1,0} / n \quad \text { and } \quad y \equiv \mu(T) \phi_{0,1},
$$



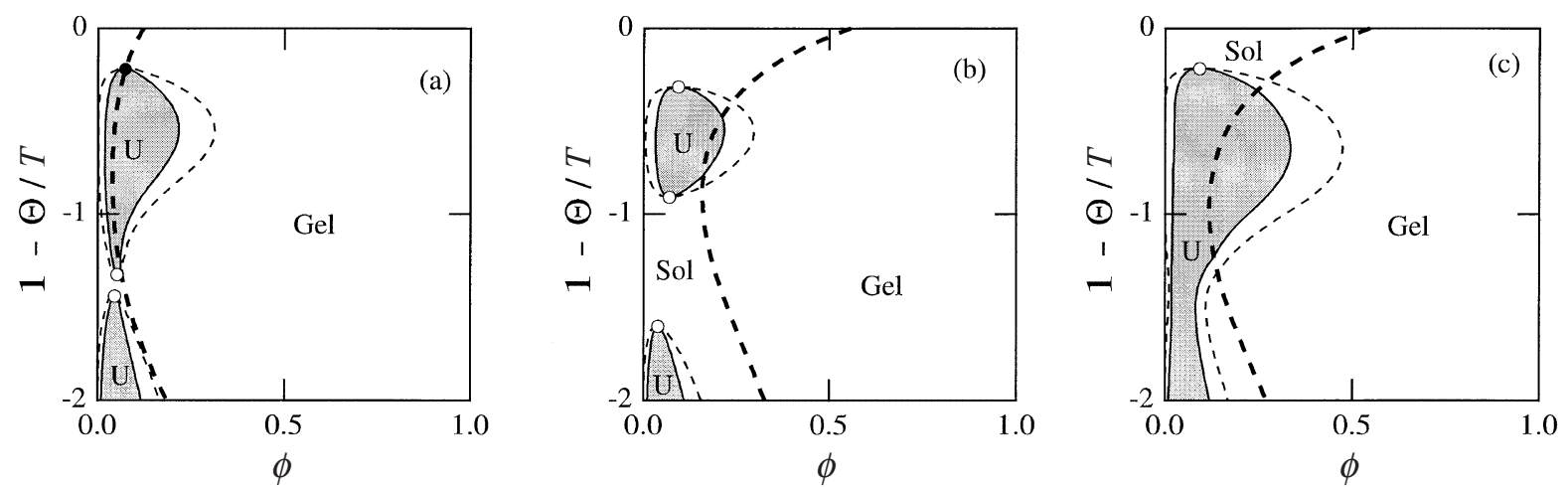

Figure 12. Phase diagrams of hydrated gels in which gelation by polymer-polymer association competes with polymer-water hydrogen bonding. Sol/gel transition lines (broken lines), binodals (solid lines) and spinodals (dotted lines) are shown. (a) For weak polymer-polymer association, closed-loop phase separation region coexists with a low-temperature phase separation dome. There is no interference between gelation and phase separation. (b) For intermediate association, sol/gel trantion line crosses the phase separation loop near the LCST of the loop. This type of LCST interfered by high-temperature gelation is often observed in water-soluble polymers. (c) For strong association, phase separation loop and dome merge and sol/gel transition line runs through it.

each corresponding to the number density of the isolated molecules, being acompanied by the temperature shift factor $\lambda$ or $\mu$. Here, temperature shift factors are given by the association constants. When there is no polymer-water coupling, i.e., $\mu=0$, eq 85 reduces to the one we obtained in the study of thermoreversible gels. ${ }^{10-12,93}$ To the contrary, when there is no polymerpolymer coupling, i.e., $\lambda=0$, eq 85 reduces to the relation we obtained in the study of closed-loop miscibility gaps of aqueous polymer solutions. ${ }^{33}$

In the following numerical calculation of the phase diagrams we use

$$
\lambda(T)=\lambda_{0} \exp \left(-\gamma_{1} \tau\right) \quad \text { and } \quad \mu(T)=\mu_{0} \exp \left(-\gamma_{2} \tau\right),
$$

by splitting the standard free energy changes into the entropy part and the energy part, where $\gamma_{1} \equiv\left|\Delta \epsilon_{1}\right| / k_{B} \Theta$, $\Delta \epsilon_{1}(<0)$ being the polymer-polymer binding energy, is the bond strength relative to the thermal energy. The polymer-water bond $\gamma_{2}$ is similarly defined. Effect of the entropy change on bonding is included in the prefactors.

Figures 12 (a) (c) show how the sol/gel transition line comes closer to the miscibility gap and interferes with phase separation as the polymer-polymer association constant is increased. ${ }^{106}$ Broken lines show sol/gel transition, solid lines binodals and dotted lines spinodals. The parameters $\gamma_{1}$ and $\gamma_{2}$ are fixed at 3.5, and $\lambda_{0}$ is changed from figure to figure $\left(\lambda_{0}=0.001\right.$ in (a), $=0.01$ in (b) and $=0.1$ in (c)). Other parameters are fixed at $n=f=100, \mu_{0}=0.05$, and $\psi_{1}=1$. As $\lambda_{0}$ becomes larger, two miscibility gaps come closer to each other and the sol/gel line shifts to low concentration region at intermediate temperatures. It does not, however, move so much at lower temperatures. This is caused by the dominance of hydration at low tem- peratures which prevents polymers from forming a gel. As the temperature becomes higher, some of the hydrating solvent molecules are detached off the chains, and the chance to form direct bonds becomes higher. The dominance of hydration at low temperatures thus results in gelation on heating. The slope of the osmotic compressibility is discontinuous across the sol/gel transition line, so that the transition is regarded as the third order phase transition.

Figure 12 (b) corresponds to what we see in the experiments on cellulose in water (Figure 4 of the ref 85) The upper half of the miscibility loop is beyond the range of the experimental observation. The sol/gel line intersects the binodal from below. The intersection, though upside down, should be regarded as a tricritical point (TCP) because it is the point where a continuousorder sol/gel transition line changes into the first-order binodal line. ${ }^{61,62}$ There is no lower critical solution point in this diagram. Instead a new tricritical phenomena are expected around the TCP.

\section{Multicomponent Gelation}

In biological and medical science, thermoreversible gels consisting of more than two species of polymers are very important. For example, it has been suggested that the repeated sol/gel transition of actin, controlled by actin-binding protein (ABP), drives motions of individual biological cells. ${ }^{107}$ In this ternary system (actin, $\mathrm{ABP}$ and water), $\mathrm{ABP}$ works as a cross-linker of the actin filaments. In the food industry, biopolymer mixtures in which either single or multiple ingredients form networks have many important applications, and have been the focus of intensive experimental study. ${ }^{108,109}$ For example, Clark et al. ${ }^{108}$ studied composite aqueous gels consisting of thermoreversible cold-setting gelling 
components such as agarose and gelatin by electronand optical microscopy. They reported that their micrographs appeared quite similar to those observed in a number of synthetic interpenetrating networks (referred to as IPNs) ${ }^{110,111}$, and showed phase separation into the two polymer networks with possible phase inversion at a certain mixture composition. Durrani et al. ${ }^{112,113}$ derived a phase diagram for the ternary amylopectin-gelatin- $\mathrm{D}_{2} \mathrm{O}$ mixture in the sol state by the use of Fourier transformed infrared microspectroscopy.

Formation of mixed networks may also be used to modify the rheological properties of aqueous polymer solutions. For instance, quite recently it was experimentally demonstrated ${ }^{114}$ that the viscosity of mixtures of the two species of polymers, $\operatorname{poly}(\mathrm{N}$ isopropylacrylamide) and hydrophobically modified poly(sodium acrylate) in aqueous solution becomes several orders of magnitude higher than is achieved without hydrophobic modification. Possible mechanism of heteropolymer cross-linking between the hydrophobes on the different species, followed by network formation by the hydrophobic aggregation of molecules, was proposed..$^{14}$

To study thermoreversible mixed gels, we consider a mixture of A-chains and B-chains, each carrying $f$ functional and $g$-functional groups. When either of the functionalities exceeds three, a cluster grows to macroscopic dimensions as soon as a threshold in the concentration is reached. ${ }^{115}$ Above this threshold a network made up of the two components is formed. The strength of the bonds can be expressed by the three association constants eq 23. By the relative strength of these constants, let us now classify the types of association into the following fundamental three categories:

(i) Interpenetrating networks (IPNs) - Polymer chains A and B are cross-linked within the same species, but do not form bonds between different species, i.e., $\lambda_{\mathrm{AB}}=0$. We refer to this case as $\mathrm{A} \cdot \mathrm{A} / \mathrm{B} \cdot \mathrm{B}$.

(ii) Alternating networks (ANs) - Cross-links are allowed only between different species, i.e., $\lambda_{\mathrm{AA}}=$ $\lambda_{\mathrm{BB}}=0$. We refer to this case as A.B. Because the clusters (of finite or infinite size) formed are multi-block copolymers, the system may undergo microphase separation. Hence, macrophase separation, microphase separation and gelation interfere with each other.

(iii) Randomly mixed networks (RMNs) - If the strengths of associative forces in the three combinations $\mathrm{AA}, \mathrm{BB}$, and $\mathrm{AB}$ are all of the same order, cluster formation progresses randomly. The resul- tant networks can be regarded as macroscopic random block-copolymers.

Networks belonging to category (i) are defined as a non-bonded, but unseparable, combination of the two different polymer networks. As a consequence of a complex dynamic balance between the two opposite tendencies, i.e., association and phase separation, synthesis of IPNs can produce materials ranging from molecularly homogeneous to microscopically phaseseparated with phase domains of various sizes, and this provides a unique method of controlling the morphology and mechanical properties. The chemistry and physics of IPNs have been extensively reviewed. ${ }^{110,111}$ There are two main methods of synthesizing them: simultaneous IPN (sim-IPN) and sequential IPN (seqIPN) formation. In sim-IPN formation, functional monomers (or primary chains) of both species A and $\mathrm{B}$ are mixed together and polymerized. In seq-IPN, a polymer network of species A is synthesized, and functional monomers B are swollen into the network and polymerized. In the present study we consider physically cross-linked networks in which junctions are formed by non-covalent associative forces, and focus our attention on the sim-IPN. In such a weakly crosslinked IPN, polymers can reach equilibrium by the reorganization (creation and annihilation) of the network junctions in the course of phase separation, and, under certain conditions, the possibility arises that a molecularly homogeneous phase comprised of two unseparable (on a time scale shorter than the junction lifetime) networks will occur.

Mixtures belonging to category (ii) form coterminously cross-linked networks when one of the components is a telechelic polymer carrying functional groups on both of its ends. ${ }^{12}$ In the extreme case where this cross-linker is the solvent molecule itself, these networks are physical gels whose junctions are made up of complexes involving solvent molecules. It was suggested from differential scanning calorimetry data 4,116 that, atactic polystyrene forms thermoreversible gels in the solvent carbon disulfide by forming solvent complexes at the network junctions, the sol/gel transition temperature exhibiting a peak as a function of the polymer concentration.

Associating polymers belonging to category (iii) form gels consisting of randomly connected twocomponent networks coexisting with the independent one-component pure gel networks. Typical examples studied so far are gelatin/agarose mixed gels in which the solution gels in a concentration region below the gelation concentration of each gelling component. ${ }^{118}$

Figures 13(a), (b) show the calculated phase diagrams ${ }^{20}$ of alternately cross-linked networks in a sym- 

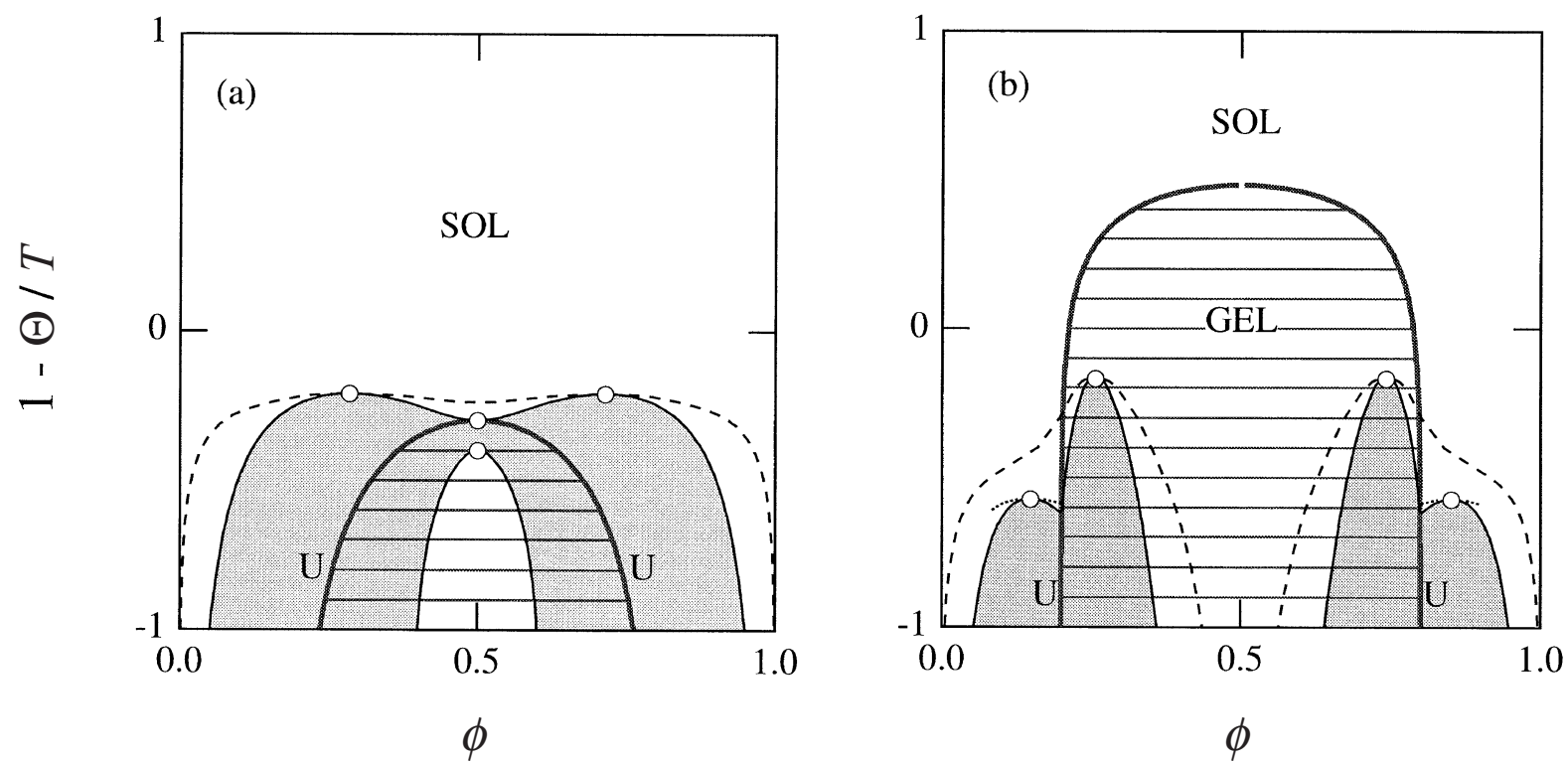

Figure 13. Phase diagrams of a alternately cross-linked network in the symmetric case (Flory's treatment); $n_{\mathrm{A}}=n_{\mathrm{B}}=10, f=g=3$, $\lambda_{0}=1.0$. Association constants $\gamma$ is changed with other parameters being fixed: (a) $\gamma=1.9926$, (b) $\gamma=5.0$. The sol/gel transition lines (thick solid lines), binodal (thin dashed line) and spinodal (thin solid line) are shown. The postgel region is indicated by thin horizontal lines. The shaded regions indicated by letter $U$, are unstable regions. The open circles show critical solution points. The gel region lies inside the spinodal for small values of association constant as in (a), but in the case of strong association as shown in (b), the miscibility gap splits into two separate pieces, and the gel region is stabilized. The alternating network works as the solvilizer in this case due to their amphiphilic nature. The critical points stay inside the gel region so that phase separation into two gels with different concentrations, and hence different cross-link density, is possible.

metric case $\left(n_{\mathrm{A}}=n_{\mathrm{B}}=10, f=g=3, \lambda_{0}=1.0\right)$. The dimensionless binding energy $\gamma$ between the functional groups on A-chains and B-chains is different. Thick solid lines show the sol/gel transition lines, thin dashed lines binodal, thin solid lines spinodal, and white circles the critical solution point. The gel region is indicated by the horizontal lines, and the unstable region (U) is shaded. For small $\gamma$, the gel region lies inside the unstable region, so that a stable homogeneous gel is not expected. With increase in the association energy, the gel region grows and miscibility is improved near the stoichiometric concentration $\phi=0.5$ (in the symmetric case) by the existence of mixed clusters. In Figure 13 (a), the population of hetero-clusters becomes so large at low temperatures that a reentrant homogeneous phase appears, and as a result, a new critical solution point appears lying on the sol/gel transition line. With further increase in the association energy, two critical solution points lying at $\phi=0.5$ merge into a single one. Then, the unstable region splits into two separate areas as is shown in Figure 13 (b).

\section{Gelation Triggared by Polymer Conformational Tran- sition}

Most natural polymers undergo a conformational transition preceding gelation. Activation of the particular functional groups on a polymer chain accom- panied by a proper three dimensional conformation change is a necessary prerequisite for the interchain cross-linking. For instance, water-soluble natural polymers such as agarose and $\kappa$-carrageenan first change their conformation from the random coil state to a partially helical state, and then the helical parts aggregate to form network junctions. ${ }^{1,4,5,119,120}$ Recently, a similar two-step mechanism of gelation through coil/helix transition was confirmed for synthetic polymers with stereo-regularity. ${ }^{121,122}$ It was found that, in solutions of syndiotactic poly(methyl methacrylate) in toluene, a fast intramolecular conformational change is followed by an intermolecular association leading eventually to gelation.

Other important examples are globular proteins. Proteins such as ovalbumin, or human serum albumin, are believed to form gels after some of the intramolecular bonds in a native state are broken during denaturation, with their functional groups being exposed to the outer space, followed by intermolecular recombination of the groups. ${ }^{1}{ }^{123,124}$ A certain degree of unfolding to expose functional groups is a neccessary condition for the gelation in these examples.

To study these examples systematically, let us here classify the types of gelation in the following way:

- Intra/Inter Transition The functional groups hidden inside a polymer molecule are activated by the change of environmental conditions such 
as the temperature, polymer concentration, $\mathrm{pH}$, concentration of another component, etc., and lead to gelation by forming intermolecular bonds. Since the subchain forming a loop by intramolecular association looks like a petal in a flower, and the dissociated functional groups form interchain bridges, this transition is often referred to as Loop/Bridge transition or Flower/Bridge transition. ${ }^{75}$ In the case where functional groups are thermally activated, this type of conformational transition leads to high-temperature gels.

- Coil/Helix, Coil/Rod or Coil/Globule Transition Polymers in random coil conformation first partially form helices (or rods, globules) as the temperature is lowered, and then helices (rods, globules) aggregate into network junctions. This mechanism results in the low-temperature gelation as complex cross-linking regions are cooperatively formed by the attractive interactions. At extremely low temperatures, however, helix sequences become longer, and, as a result, the total number of helices on a chain decreases. ${ }^{126}$ The restriction in selecting helix sequences from the limited chain length, thus, tends to prevent gelation.

- Two-State Transition Each monomeric unit A along a polymer chain can take either an active state $\mathrm{A}^{*}$ or an inert state $\mathrm{A}$. The active monomeric units form cross-links of the type $\left(\mathrm{A}^{*}\right)_{k}$ with multiplicity $k(k=2,3,4, \ldots)$. This type may also lead to high-temperature gelation.

To study such systems we introduce conformational term

$$
\Delta F_{\text {conf }}=A_{\lambda} N_{\lambda}+\sum_{\mathbf{j}, \mathbf{l}}\left(\sum_{f \geq 1} A_{f} l_{f}\right) N(\mathbf{j} ; \mathbf{l})+\sum_{f \geq 1} A_{f} N_{f}^{\mathrm{G}},
$$

into our model free energy, ${ }^{117}$ where $N_{\lambda}$ is the number of inert molecules (called $\lambda$-molecules) that carry no active functional groups, $N_{\mathrm{f}}^{\mathrm{G}}$ the number of primary molecules in the gel network that carry $f$ active functional groups (called $\mu$-molecules), $A_{\lambda}$ the conformational free energy of a single $\lambda$-molecule and $A_{f}$ the same of an active molecule with $f$ active groups. The free energy required for the activation of a molecule is therefore given by

$$
\Delta A_{f} \equiv A_{f}-A_{\lambda} .
$$

The usage of the terms $\lambda$ and $\mu$ comes from equilibrium polymerization of sulfur. ${ }^{127,128}$ Equilibrium polymerization of surfur is a special case of the above intra/inter transition. A ring polymer $\mathrm{S}_{8}$ (called $\lambda$-sulfur), which is inert at room temperature, first opens its ring into a linear chain carrying reactive groups on both its ends (called $\mu$-sulfur) as the temperature is raised, and then polymerized through interchain bonding at $160^{\circ} \mathrm{C}$. Since the reaction takes place pairwisely and the functionality $f$ (number of active sites on a molecule) of $\mu$-sulfur is two, molecules form linear chains instead of three dimensional networks.

First let us consider the activation equilibrium, i.e., the equilibrium between $\lambda$-molecules and $f$-molecules in the $\mu$-state. It is given by the condition

$$
\Delta \mu_{\lambda}=\Delta \mu\left(\mathbf{j}_{0 f} ; \mathbf{l}_{0 f}\right) .
$$

On substitution of the explicit forms of the chemical potentials, we find that the volume fraction of $f$ molecules in the solution is uniquely related to the volume fraction of the $\lambda$-molecules through the equation

$$
\phi\left(\mathbf{j}_{0 f} ; \mathbf{l}_{0 f}\right)=\phi_{\lambda} \exp \left(-\beta \Delta A_{f}\right) .
$$

The excitation probability is given by

$$
\eta_{f} \equiv e^{-\beta \Delta A_{f}} .
$$

For independent excitation, $\eta_{f}=\eta_{1}^{f}$, and $\eta_{1}$ is assumed to take a form $\eta_{1}=\eta_{0} \exp \left(\gamma^{\prime} \tau\right)$ for numerical calculation.

The relation eq 70 is then rewritten as

$$
\frac{\lambda f_{\mathrm{av}}(z)}{n} \phi=z u(z),
$$

where

$$
f_{\mathrm{av}}(z) \equiv \sum_{f \geq 0} f u(z)^{f} \eta_{f} / \sum_{f \geq 0} u(z)^{f} \eta_{f},
$$

is the number-average functionality of polymer chains.

Figures 14(a), (b) show how the phase behavior changes depending upon the relative strength of the association constant and the excitation constant. These phase diagrams are calculated for trifunctional $(f=3)$ low molecular weight molecules $(n=1)$ with triple junctions $(s=3)$. Independent excitation of the functional groups is assumed. In these diagrams, solid lines show binodal, broken lines sol/gel transition, and shaded areas are unstable regions. When the association constant is large as in Figure 14 (a), the solution exhibits UCST type phase separation intersecting with the low-temperature sol/gel transition line at the top of the phase separation region. With decrease in the strength of association, or increase in the excitation constant, association in the low temperature region becomes less favorable, and as a result, the lower part of a sol/gel line tends to shift to higher concentration region. The unstable region around the sol/gel transition line move upwards following the shift of the sol/gel transition line. In the case of extremely large excitation constant as in Figure 14 (b), the gel region completely separates from 
(a)

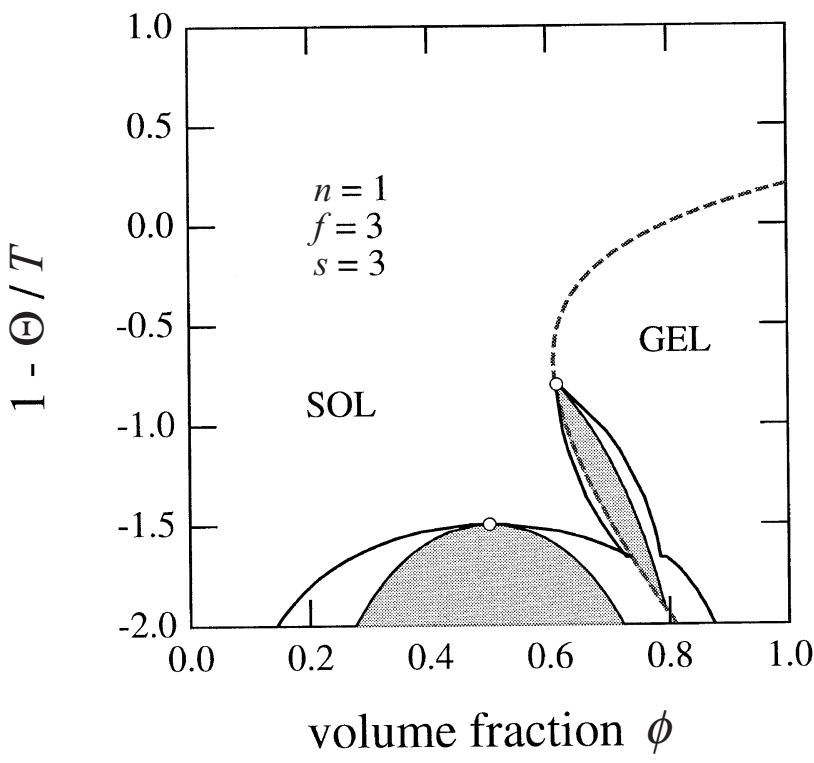

(b)

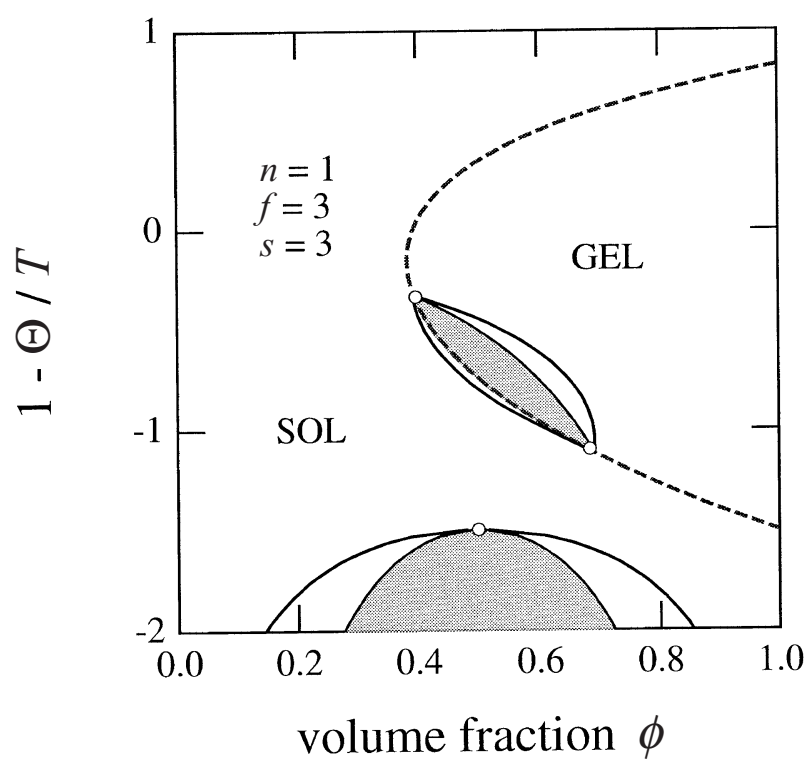

Figure 14. Phase diagrams of low molecular weight primary molecules $(n=1)$ with triple associative groups $(f=3)$. Independent excitation followed by triple association $(s=3)$ is assumed. Association constant and excitation constant are fixed at (a) $\lambda_{0}=0.73, \gamma=$ $2.50, \eta_{0}=1.00, \gamma^{\prime}=2.80$; (b) $\lambda_{0}=1.48, \gamma=2.00, \eta_{0}=1.00, \gamma^{\prime}=3.00$.

the UCST miscibility dome, because strong thermal excitation is required to activate functional groups. Extra LCST and UCST appear at the top and bottom of the high-temperature isolated two-phase region. This diagram resembles that of the equilibrium polymerization of sulfur in a solution, ${ }^{128}$ but the polymerization line is replaced by the gelation line. Tobitani and RossMurphy ${ }^{124}$ confirmed a similar gelation line in the study of heat-induced gelation in aqueous solution of globular protein (bovine serum albumin).

This study is directly applicable to thermoreversible gelation triggared by coil/helix transition of polymer chains. Details will be published elsewhere.

\section{Polymer-Surfactant Interaction}

The interactions between polymers and surfactants have been a subject of great interest. ${ }^{129,130}$ The problem was laid initially in studies of proteins associated with natural lipids, and later in the studies of their association with synthetic surfactants. More recently, interaction of water-soluble synthetic polymers such as poly(ethylene oxide) with ionic and non-ionic surfactants ${ }^{131-134}$ have attracted the interest of researchers because of the scientific and technological implications. Adding surfactants to polymer solutions with formation of polymer/surfactant complex can substantially alter the physical properties of the starting polymers. The effects can be summarized into the following four categories: (i) Conformational transition of polymers such as coil/globule transition ${ }^{135,136}$ and coil/rod transition, ${ }^{137,138}$ (ii) Expansion and shift of the phase separation region on the polymer/solvent phase plane, ${ }^{139}$ (iii) Formation of composite microphases, ${ }^{140}$ (iv) Shift of the sol/gel transition line ${ }^{141-143}$ and modification of the rheological properties. ${ }^{71,80,82}$ When polymers carry small fractions of hydrophobic groups, effects are dramatically enhanced, because the ability of surfactant binding is enhanced by the hydrophobic interaction between polymer hydrophobes and surfactant hydrophobes. A profound influence of added surfactants on the rheological properties has been reported. For example, the high-frequency plateau modulus of HEUR solution exhibits a large peak when sodium dodecyl sulfate (SDS) is added at low polymer concentrations. The peak in the modulus disappears at higher polymer concentrations. $^{71}$

To study the effect of added surfactants, we consider a model mixture of APs and low molecular-weight surfactant molecules in a solvent. ${ }^{144}$ Each polymer is assumed to carry the number $f(\geq 2)$ of associative groups along its chain, and each surfactant molecule is modeled as a low molecular weight molecule carrying a single hydrophobe connected to the hydrophilic head. Therefore, the system is a special case of the model solution treated above.

In equilibrium, hydrophobes on the polymers and on the surfactants aggregate into mixed micelles that serve as cross-link junctions. The aggregation number differs from one micelle to another. If we use the (reduced) 


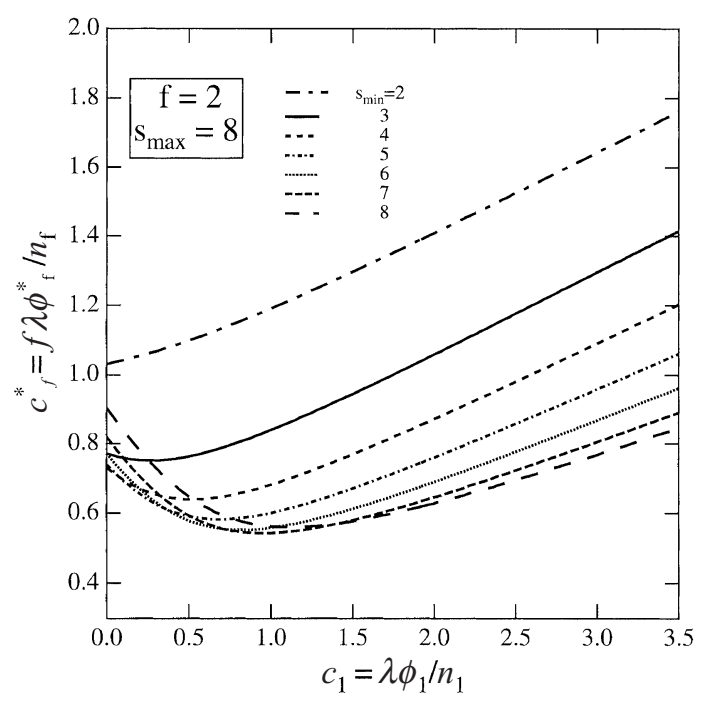

Figure 15. Polymer concentration at sol/gel transition as a function of the concentration of added surfactant. The concentration is measured in terms of the number density $f \phi / n$ of the hydrophobes times the association constant $\lambda(T)$ for each species. Minimum multiplicity $s_{\min }$ is varied from curve to curve under a fixed maximum multiplicity $s_{\max }$. While the sol/gel concentration monotonically increases with surfactant concentration for $s_{\min }=2$ (i.e., there is no lower bound in the multiplicity), there appears a minimum at a certain surfactant concentration for $s_{\min } \geq 3$ (i.e., if there is a gap below the minimum allowed multiplicity). In the latter case, gelation is promoted by the surfactant molecules, and referred to as surfactant-mediated gelation (SMG) in the text.

concentration $c_{f} \equiv \lambda(T) f \phi_{f} / n_{f}$ instead of $\phi_{f}$ for polymers and $c_{1} \equiv \lambda(T) \phi_{1} / n_{1}$ for surfactants $\left(n_{i}\right.$ being the number of elementary statistical units on the molecule of species $i$ ), the relation eq 70 can be transformed into

$$
c_{f}+c_{1}=z u(z) \text {. }
$$

Solving this relation with respect to $z$ for a given $c_{f}$ and $c_{1}$, we find $z$, and hence the conversion $\alpha$ as a function of a given temperature and concentrations of both components.

The sol/gel transition condition for our polymer/surfactant system is then explicitly given by

$$
(f-1) c_{f} z u^{\prime}(z) /\left(c_{f}+c_{1}\right) u(z)=1 .
$$

Combining this condition with the above relation and eliminating the parameter $z$, we find the sol/gel transition curve on the temperature-concentration plane.

When polymer concentration is low and the number of hydrophobes is not enough to form junctions, addition of surfactants combines the unassociated hydrophobes into forming stable junctions with aggregation number exceeding $s_{\min }$. In this situation, the surfactant works as a cross-linking agency. On the contrary, when the polymer concentration is large and many junctions are already formed, some of the polymer hydrophobes in the junctions are replaced by surfactant hydrophobes as soon as their multiplicity reaches $s_{\max }$, leading to dissociation of network junctions.

To demonstrate these opposite effects, we calculate the concentration of polymers at the sol/gel transition point as a function of the concentration of the added surfactant. ${ }^{144}$ Figure 15 shows the result for the telechelic $(f=2)$ polymers. Both polymer and surfactant concentrations are expressed in terms of the reduced concentration, the number of hydrophobes (per lattice cell) times association constant. To see the effect of the minimum multiplicity, $s_{\min }$ is varied from curve to curve, while the maximum multiplicity is fixed at $s_{\max }=8$. It is clear that the sol/gel concentration $c_{f}^{*}$ monotonically increases with the surfactant concentration for $s_{\min }=2$ (no lower bound), i.e., gelation is simply blocked by the surfactant. But if there is a forbidden region between $k=1$ (unassociated) and $k=s_{\min }$, a minimum in $c_{f}^{*}$ starts to appear. At this surfactant concentration gelation is most promoted as can be seen for $s_{\min } \geq 3$. The surfactant concentration at which $c_{f}^{*}$ becomes minimum is referred to as the surfactantmediated gelation (SMG) point. It increases as the minimum multiplicity becomes larger.

We next study the effect of added surfactants on the dynamic mechanical moduli of AP solution. In the experiment of HEUR/SDS system, ${ }^{71}$ addition of surfactant results in several effects. The moduli can no longer be described by the simple Maxwell element with a single relaxation time, but a shoulder appears on the loss modulus at high frequencies. The high frequency plateau in the storage modulus reveals nonmonotonic dependence on the SDS concentration. ${ }^{71,82}$ At low polymer concentration, it initially rises to a peak and then decreases monotonically, falling eventually to zero at higher SDS concentration. With increase in the polymer concentration, the height of the peak in the storage modulus decreases and its position shifts to lower SDS concentration. Above a certain polymer concentration, the peak disappears.

The plateau value of $G^{\prime}(\omega)$ is expected to be proportional to $v_{\mathrm{eff}}\left(c_{1}\right) k_{\mathrm{B}} T$ because the network topology does not alter within the measurement timescale $\omega^{-1}$, which is shorter than the average lifetime $\tau_{\times}$of a junction. Therefore, we can study the elastic moduli by counting the number of elastically effective chains as a function of the concentration of surfactant. Figure 16 shows the number $v_{\text {eff }}\left(c_{1}\right)$ plotted against the surfactant concentration $c_{1}$ (divided by the polymer concentration $c$ ) for $f=2$. The number is normalized by the value $v_{\text {eff }}(0)$ under the absence of the surfactant. This ratio gives the relative amplitude $G^{\prime}\left(c_{1}\right) / G^{\prime}(0)$ of the high frequency plateau value in the storage modulus. The 


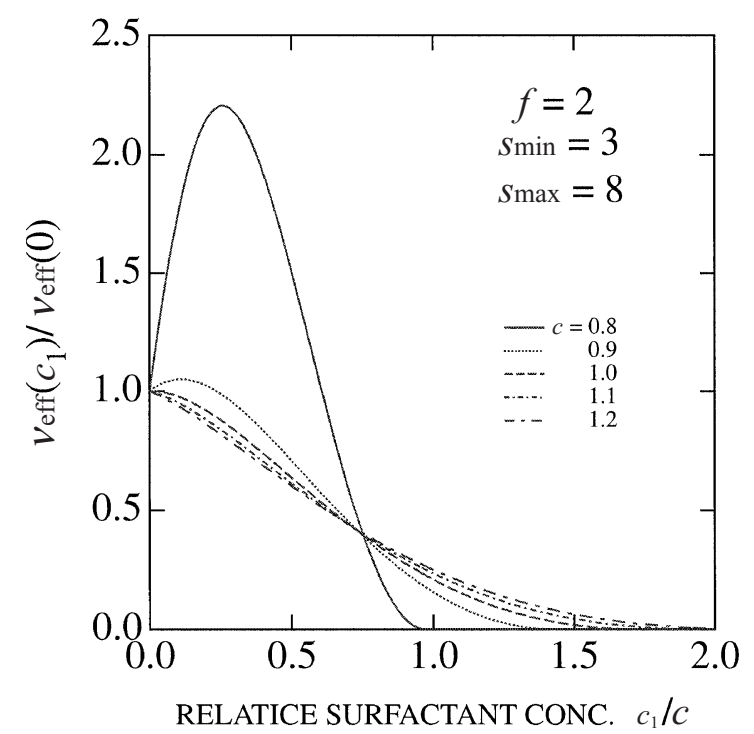

Figure 16. The number of elastically effective chains plotted against the ratio of the surfactant concentration and the polymer concentration. The polymer concentration is varied from curve to curve. At low polymer concentrations, the number of effective chains, and hence the high frequency modulus, shows a peak at SMG surfactant concentration and then decreases. But at higher polymer concentration, it monotonically decreases. The effective chains disappear at a high surfactant concentration at which solution turns into sol. Note that all curves cross at a single point.

multiplicity of a junction is varied from 3 to 8 . The polymer concentration $c_{f=2}$ is changed from curve to curve. As expected, the curves of the number of elastically effective chains for low polymer concentrations first rise to a peak and then monotonically decrease to zero where the gel network is broken into sol by the surfactant. For higher polymer concentrations, however, the curves do not show any peak, because junctions are well developed without surfactant molecules for such polymer concentrations and the added surfactant merely destroys junctions. These calculations reproduce, at least qualitatively, the experimental data on such as HEUR/SDS reported by Annable et al. ${ }^{71}$

\section{THEORY OF TRANSIENT NETWORKS}

In this section we concern dynamic properties of the thermoreversible networks to study enhanced viscoelasticity due to the presence of the temporal junctions that are created and destroyed under thermal fluctuation and/or external stress.

The first systematic study on thermoreversible networks was the transient network theory developed by Green and Tobolsky ${ }^{145}$ in which stress relaxation in rubber-like polymer networks was treated by the kinetic theory of rubber elasticity suitably extended so as to allow for the creation and annihilation of junctions during the network deformation. In order to ensure wider range of applicability, some arbitrary assumptions in their theory were later removed by Lodge ${ }^{146}$ and Yamamoto ${ }^{147}$ with the intention to apply it to the entangled melts ${ }^{148,149}$ (rather than the reversible networks). In their studies, to describe the observed viscoelastic properties of ordinary polymer melts, localized entanglements were regarded as the temporal junctions which can be created and destroyed during the macroscopic deformation. Because of the lack of the detailed knowledge about molecular mechanism of junction generation (onset of entanglements), their theories, however, remain semiphenomenological.

Here, we propose a new type of transient network theory ${ }^{150,151}$ to ensure a molecular picture which is suitable to describe real networks in which crosslinks are actually generated and destroyed. We find a specific form of the junction generation function, which is dependent on the number of active chains present at the moment of linkage - and hence dependent on the macrodeformation at the same moment.

\section{Three Fundamental Timescales}

Multiple junctions formed by weak associative interaction can break and recombine with a finite average lifetime $\tau_{\times}$. To describe creation and annihilation we introduce ${ }^{150}$ a model network made up of polymers of uniform molecular weight $M$ (or the number $n$ of statistical units) carrying associative functional groups at their both chain ends (telechelic polymer). We focus our attention specifically on the unentangled regime where $M$ is smaller than the entanglement molecular weight $M_{\mathrm{e}}$, so that each chain obeys Rouse dynamics ${ }^{148,149}$ modified by mutual end-association. For the entangled regime where $\tau_{\times}$is comparable to the entanglement release time, phenomenological theory to study observed two-step stress relaxation was proposed. $^{152}$

In the following, we assume the lifetime of a junction is sufficiently long so that it is well separated from the Rouse relaxation spectrum, the longest time of which is given by $\tau_{R} \equiv \zeta a^{2} n^{2} / 3 \pi^{2} k_{\mathrm{B}} T$, where $\zeta$ is the friction coefficient of a monomer with the size $a$.

We consider a time interval $\mathrm{d} t$ smaller than $\tau_{\times}$but still larger than $\tau_{\mathrm{R}}$. Under a macroscopic deformation $\hat{\lambda}(t)$ given to the network, either end of a chain, being stretched above a critical length, disengages from the junction and the chain relaxes to a Gaussian conformation, whilst some of the free dangling ends recapture the junctions in their neighborhood (Figure 17). Since the stress is transmitted only through the chains whose both ends are connected to the network junctions, we call these chains elastically effective (or active) chains 


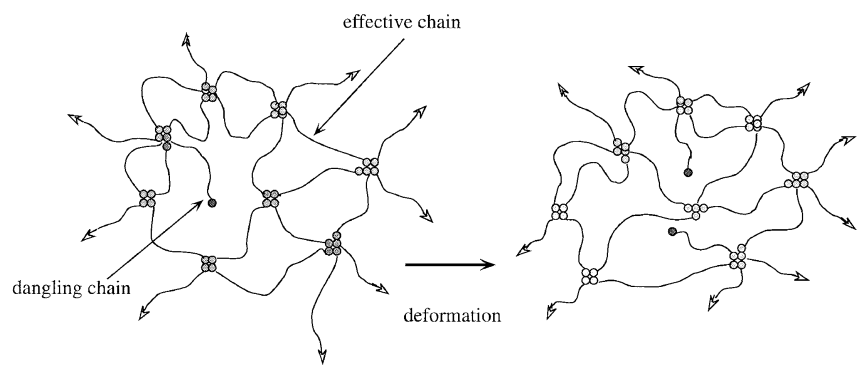

Figure 17. Chain disengagement and recombination in the transient network under a macroscopic deformation. The network is made up of telechelic polymers with associative groups at their chain ends.

as in the preceding sections.

\section{Transient Network Model}

Let $N$ be the total number of polymer chains in a unit volume, and let $F(\mathbf{r}, t) \mathrm{d} \mathbf{r}$ be the number of active chains at time $t$ whose end-to-end vector $\mathbf{r}$ falls on a small region $\mathrm{d} \mathbf{r}$ around $\mathbf{r}$. The total number of active chains at time $t$ is given by $v(t) \equiv \int F(\mathbf{r}, t) \mathrm{d} \mathbf{r}$. By counting the number of active chains that are destroyed and created in the interval $\mathrm{d} t$ under the deformation $\hat{\lambda}(t)$, it is shown ${ }^{150,151}$ that the time development of the distribution is governed by the equation

$$
\begin{aligned}
& F(\mathbf{r}, t) \mathrm{d} \mathbf{r}=\Theta\left(\mathbf{r}, t ; \mathbf{r}_{0}, 0\right) F\left(\mathbf{r}_{0}, 0\right) \mathrm{d} \mathbf{r}_{0} \\
& \quad+p \int_{0}^{t} \mathrm{~d} t^{\prime} \Theta\left(\mathbf{r}, t ; \mathbf{r}^{\prime}, t^{\prime}\right)\left[N-v\left(t^{\prime}\right)\right] f_{0}\left(\mathbf{r}^{\prime}\right) \mathrm{d} \mathbf{r}^{\prime},
\end{aligned}
$$

where

$$
\Theta\left(\mathbf{r}, t ; \mathbf{r}^{\prime}, t^{\prime}\right) \equiv \exp \left[-\int_{t^{\prime}}^{t} \beta\left(\mathbf{r}_{t^{\prime \prime}, t^{\prime}}\right) \mathrm{d} t^{\prime \prime}\right],
$$

is the probability for an active chain created at a certain time $t^{\prime}$ in the past to remain active until the present time $t$. The function $\beta(\mathbf{r})$ gives the probability per unit time for an active chain to disengage from the junction and is called chain breakage rate. The parameter $p$ in the second term is the probability per unit time for a dangling end to capture a junction (called chain recombination rate). We have assumed that end-to-end vectors are mapped affinely to the macroscopic deformation so that we have $\mathbf{r}_{t^{\prime \prime}, t^{\prime}}=\hat{\lambda}\left(t^{\prime \prime}\right) \cdot \hat{\lambda}\left(t^{\prime}\right)^{-1} \cdot \mathbf{r}^{\prime}$. etc. The equilibrium distribution $f_{0}(\mathbf{r})$ of the end-to-end vector is assumed to take the conventional Gaussian form with the average square distance $\left\langle r^{2}\right\rangle_{0}=n a^{2}$.

Upon integration over $\mathbf{r}$, the total number $v(t)$ per unit volume of the active chains is found to obey an integral equation

$$
v(t)=v_{i n}(t)+p \int_{0}^{t} \zeta\left(t ; t^{\prime}\right)\left[N-v\left(t^{\prime}\right)\right] \mathrm{d} t^{\prime},
$$

where the function $\zeta\left(t ; t^{\prime}\right)$ defined by

$$
\zeta\left(t ; t^{\prime}\right) \equiv\left\langle\exp \left[-\int_{t^{\prime}}^{t} \beta\left(\mathbf{r}_{t^{\prime}, t^{\prime \prime}}\right) \mathrm{d} t^{\prime \prime}\right]\right\rangle_{0}
$$

in terms of the chain breakage rate $\beta(\mathbf{r})$ gives the average probability for an active chain created at time $t^{\prime}$ to remain active until time $t$, and is named chain survival function. The symbol $\langle\ldots\rangle_{0}$ indicates an average over Gaussian distribution.

The stress tensor corresponding to a given deformation $\hat{\lambda}(t)$ is readily derived from the total free energy $\Phi(t) \equiv \int \mathrm{d} \mathbf{r} \phi(\mathbf{r}) F(\mathbf{r}, t)$ where

$$
\phi(r)=\frac{3 k_{\mathrm{B}} T}{2 n a^{2}} r^{2}
$$

is the free energy stored in an effective chain with endto-end distance $r$. Substituting the integral form for $F(\mathbf{r}, t)$ and taking the derivative by $\hat{\lambda}(t)$, we find

$$
\hat{\Sigma}(t)=\hat{\Sigma}_{i n}(t)+p \int_{0}^{t} \hat{\sigma}\left(t ; t^{\prime}\right)\left[N-v\left(t^{\prime}\right)\right] \mathrm{d} t^{\prime}-P \hat{1},
$$

for the time development of the stress tensor, where

$$
\hat{\Sigma}_{i n} \equiv \int\left(\frac{\phi^{\prime}(r)}{r}\right) \mathbf{r}^{\mathrm{T}} \mathbf{r} \Theta\left(\mathbf{r}, t ; \mathbf{r}_{0}, 0\right) F\left(\mathbf{r}_{0}, 0\right) \mathrm{d} \mathbf{r}_{0}
$$

is the stress sustained by the initially active chains, and

$$
\hat{\sigma}\left(t ; t^{\prime}\right) \equiv \int\left(\frac{\phi^{\prime}(r)}{r}\right) \mathbf{r}^{\mathrm{T}} \mathbf{r} \Theta\left(\mathbf{r}, t ; \mathbf{r}^{\prime}, t^{\prime}\right) f_{0}\left(\mathbf{r}^{\prime}\right) \mathrm{d} \mathbf{r}^{\prime}
$$

is the time propagator for the stress survival. Here, the symbol ${ }^{\mathrm{T}} \mathbf{r}$ shows the transposed vector of $\mathbf{r}$. The last term of eq 102 gives the isotropic pressure.

\section{Chain Breakage Rate}

Let us next consider a realistic form of $\beta(r)$. We focus our attention on a single active chain. One end reactive group dissociates from the junction either by its own thermal motion or by being pulled by the tension exerted from the chain connected to it. If the reactive group is isolated-that is, has no chain connected to it-, it must overcome the potential barrier $W$ to dissociate. The probability for this process to occur is proportional to $\exp \left(-W / k_{\mathrm{B}} T\right)$, and hence the dissociation rate per unit time takes the form

$$
\beta_{0}=\omega_{0} e^{-W / k_{\mathrm{B}} T},
$$

where $\omega_{0}$ is the natural frequency of thermal vibration of the reactive group in the isolated state, or equivalently, the average number of times it tries to climb up the potential barrier by thermal motion. This frequency is a microscopic measure of the time and should take a typical value on the order of $10^{8}-10^{9} \mathrm{~s}^{-1}$ in ordinary 
circumstances. As the reactive group is attached on the chain end, however, it is under the influence of the additional force transmitted along the chain. This force $f_{\mathrm{r}}$ works in the direction of the other end of the chain. Its absolute value is given by

$$
f_{\mathrm{r}}=\frac{\partial \phi}{\partial r}=\frac{3 k_{\mathrm{B}} T}{n a^{2}} r
$$

for a Gaussian chain, where $\phi(r)$ is the free energy stored in the chain. Being pulled by this force, the potential barrier for A to dissociate is effectively reduced to $W-f_{\mathrm{r}} \cdot a$, so that the probability of the chain breakage per unit time is enhanced to the value

$$
\beta(r)=\omega_{0} \exp \left[-\left(W-f_{\mathrm{r}} a\right) / k_{\mathrm{B}} T\right] \equiv \beta_{0} e^{\kappa r},
$$

where $\beta_{0}$ is given by eq 105 as before and $\kappa \equiv 3 / n a$ is a small parameter which depends on the molecular weight of the polymer chain, and $\tau_{\times}=\beta_{0}^{-1}$ is the relaxation time $\tau_{\times}$given above. This form reflects the activation process: the relaxation time depends exponentially on the temperature. Temperature dependence of the dominant time scale is one of the most important differences between temporalily crosslinked networks and uncrosslinked polymer melts. ${ }^{148,149}$ (In the latter systems, both Rouse relaxation time and the reptation time are virtually proportional to $T^{-1}$ apart from the indirect dependence through the friction coefficient).

\section{Equilibrium Distribution}

In the following we consider an aged system-that is, a network which is kept quiescent with no external perturbation for a sufficiently long time, so that it remains intact in an equilibrium state. Since $\partial F / \partial t=0$, eq 97 gives a balance

$$
p\left(N-v_{0}\right) f_{0}(\mathbf{r})=\beta(\mathbf{r}) F(\mathbf{r}, 0)
$$

for the initial distribution, where $v_{0} \equiv \int F(\mathbf{r}, 0) \mathrm{d} \mathbf{r}$ is the number of active chain in the initial equilibrium. This balancing condition requires that $F(\mathbf{r}, 0)$ must be proportional to $f_{0}(\mathbf{r}) / \beta(\mathbf{r})$. Correct normalization gives $F(\mathbf{r}, 0)=v_{0} f(\mathbf{r}, 0)$, where

$$
f(\mathbf{r}, 0) \equiv \frac{f_{0}(\mathbf{r})}{\zeta_{0} \beta(\mathbf{r})}
$$

with

$$
\zeta_{0} \equiv \int \frac{f_{0}(\mathbf{r})}{\beta(\mathbf{r})} \mathrm{d} \mathbf{r}
$$

being the normalization constant. The initial distribution is therefore not Gaussian if the chain breakage rate $\beta$ depends on the end-to-end distance. Substituting this form for $f$ into eq 108 , we find

$$
v_{0}=\frac{p \zeta_{0}}{1+p \zeta_{0}} N
$$

Among the total $N$ of the chains forming the network, the fraction $p \zeta_{0} /\left(1+p \zeta_{0}\right)$ turns out to be active.

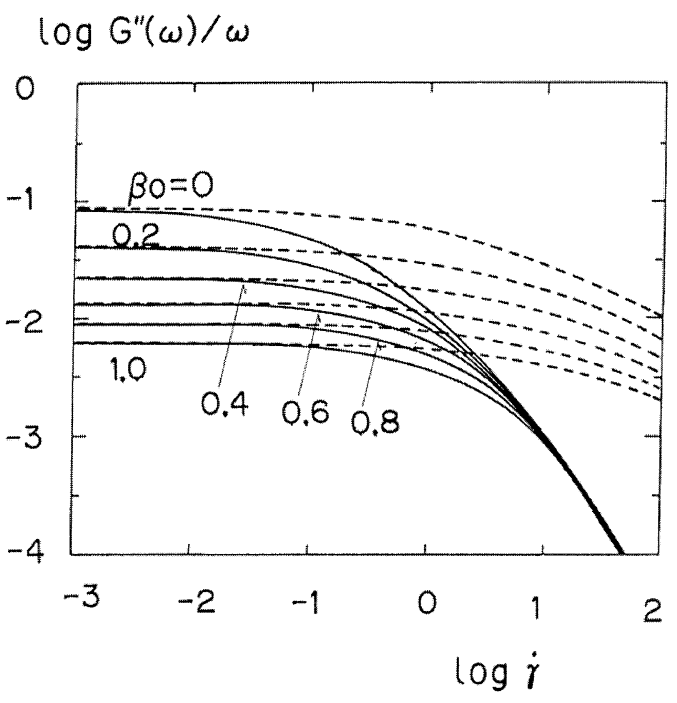

Figure 18. Comparison between stationary nonlinear viscosity(broken lines) and linear frequency-dependent viscosity (solid lines). Cox-Merz rule does not hold at high shear rate.

\section{RHEOLOGICAL PROPERTIES OF THERMOREVERSIBLE NETWORKS}

\section{Stationary Non-Linear Viscosity and Shear Thickening}

Let us first study stationary solution of the fundamental equation under shear flow with the shear rate $\dot{\gamma}$. The deformation tensor $\hat{\lambda}(t)$ takes the form

$$
\hat{\lambda}(t)=\left(\begin{array}{ccc}
1 & \dot{\gamma} t & 0 \\
0 & 1 & 0 \\
0 & 0 & 1
\end{array}\right) .
$$

When the chain breakage rate is independent of $r$ and given by $\beta(r)=\beta_{0}$, the network has a single relaxation time $\tau_{\times}=\beta_{0}{ }^{-1}$ representing the average bound time of a hydrophobe. This simplest model for $\beta(r)$ was first considered by Green and Tobolsky, ${ }^{145}$ so that in the following we shall refer to it as Green-Tobolsky limit (or GT limit). Although it is physically unrealistic, a great deal can be inferred from such an extreme case concerning the viscoelastic properties of the transient network. The steady state number of active chains is independent of the deformation rate. It remains the same as the equilibrium value under no external forces. The shear viscosity becomes $\eta(\dot{\gamma})=v_{0} k_{\mathrm{B}} T / \beta_{0}=G_{0} \tau_{\times}$, where $G_{0} \equiv v_{0} k_{\mathrm{B}} T$ is the linear elastic modulus of an affine network. Here, $v_{0} \equiv p N /\left(p+\beta_{0}\right)$ is the number of active chains in an equilibrium state. There is neither shear thinning nor shear thickening in the GT limit.

In Figure 18, we compare theoretically calculated stationary nonlinear viscosity $\eta(\dot{\gamma})$ with linear frequency-dependent viscosity $\eta(\omega)$ (discussed in the following sections) for the chain breakage rate eq 107. In usual polymer solutions, these two agree (so called 
Cox-Merz rule), but for thermoreversible transient networks they separate at high shear rates, thus indicating brealdown of Cox-Merz rule. Our simple transient network model, however, does not lead to shear thickening.

\section{Linear Response and Dynamic Mechanical Modulus}

In order to derive the dynamic mechanical moduli, let us consider a small oscillatory deformation given by the deformation tensor $\hat{\lambda}(t)=1+\hat{\epsilon} \sin \omega t$. For example, the small tensor $\hat{\epsilon}$ is given by

$$
\hat{\epsilon}=\left(\begin{array}{lll}
0 & \epsilon & 0 \\
0 & 0 & 0 \\
0 & 0 & 0
\end{array}\right)
$$

for a shear deformation. The end-to-end distance at time $t^{\prime \prime}$ is then expanded as

$$
\left|\hat{\lambda}\left(t^{\prime \prime}\right) \cdot \hat{\lambda}\left(t^{\prime}\right) \mathbf{r}\right|=r+h(\mathbf{r})\left(\sin \omega t^{\prime \prime}-\sin \omega t^{\prime}\right)+O\left(\epsilon^{2}\right),
$$

where the function $h(\mathbf{r})$ of order $\epsilon$ is defined by $h(\mathbf{r}) \equiv$ $\left({ }^{\mathrm{T}} \mathbf{r} \cdot \hat{\epsilon} \cdot \mathbf{r}\right) / r$.

Substituting this series expansion into eq 102 and expanding the result again in powers of $\epsilon$, we find

$$
\Sigma_{x y}(t)=\epsilon k_{\mathrm{B}} T v_{0}\left[g_{1}(\omega) \sin \omega t+g_{2}(\omega) \cos \omega t\right]+\cdots,
$$

where new functions $g_{i}(\omega)$ are explicitly given by

$$
\begin{aligned}
& g_{1}(\omega) \equiv \frac{1}{\zeta_{0}\left\langle r^{2}\right\rangle_{0}}\left\langle\frac{\omega^{2} r^{2}}{\beta(r)\left[\omega^{2}+\beta(r)^{2}\right]}\left[1-\frac{2 r \beta(r) \beta^{\prime}(r)}{5\left[\omega^{2}+\beta(r)^{2}\right]}\right]\right\rangle_{0}, \\
& g_{2}(\omega) \equiv \frac{1}{\zeta_{0}\left\langle r^{2}\right\rangle_{0}}\left\langle\frac{\omega r^{2}}{\omega^{2}+\beta(r)^{2}}\left[1+\frac{\left[\omega^{2}-\beta(r)^{2}\right] r \beta^{\prime}(r)}{5 \beta(r)^{2}\left[\omega^{2}+\beta(r)^{2}\right]}\right]\right\rangle_{0} .
\end{aligned}
$$

On the basis of this fundamental equation, we find that the storage modulus $G^{\prime}(\omega, T)$ and the loss modulus $G^{\prime \prime}(\omega, T)$ for shear deformation of the network is given by

$$
\begin{aligned}
& G^{\prime}(\omega, T)=v_{e}(T) k_{\mathrm{B}} T g_{1}(\omega, T), \\
& G^{\prime \prime}(\omega, T)=v_{\mathrm{e}}(T) k_{\mathrm{B}} T g_{2}(\omega, T),
\end{aligned}
$$

where $v_{\mathrm{e}}(T)$ is the number of elastically effective chains in the network at temperature $T$.

In the GT limit, our model gives conventional Maxwell fluid with a single relaxation time.

Upon substitution of eq 107 into the moduli, we find that a modulus-frequency curve at any temperature $T$ can be superimposed onto a single master curve at the reference temperature $T_{0}$ by proper horizontal and vertical shift. Specifically we have

$$
\frac{G(\omega)}{v_{\mathrm{e}}\left(T_{0}\right) k_{\mathrm{B}} T_{0}} b_{\mathrm{T}}=g\left(\frac{\omega}{\beta_{0}\left(T_{0}\right)} a_{\mathrm{T}}\right)
$$

for both $G^{\prime}$ and $G^{\prime \prime}$, where

$$
a_{\mathrm{T}} \equiv \beta_{0}\left(T_{0}\right) / \beta(T)=\exp \left[-\frac{W}{k_{\mathrm{B}}}\left(\frac{1}{T_{0}}-\frac{1}{T}\right)\right]
$$

is the frequency (horizontal) shift factor ( $W$ being the energy part in $\Delta F$ ), and

$$
b_{\mathrm{T}} \equiv v_{\mathrm{e}}\left(T_{0}\right) k_{\mathrm{B}} T_{0} / v_{\mathrm{e}}(T) k_{\mathrm{B}} T
$$

is the mudulus (vertical) shift factor. From $a_{\mathrm{T}}$, or the peak position of the loss modulus, we find the free energy barrier $W$ of activation, and from $b_{\mathrm{T}}$ or the high frequency plateau value of the storage modulus, we find the number $v_{\mathrm{e}}$ of elastically effective chains.

\section{Transient Flows}

In this section, we study time-dependent rheological properties for the defomation $\hat{\lambda}(t)$ that varies with time. In some experiments, a uniform deformation is given to the sample at certain time and kept at a constant value. The stress suddenly rises, but soon decay to the initial equilibrium level. In more detailed experiments, a shear flow with a constant shear rate is suddenly started. The stress gradually increases and reaches a maximum value, and then decays to the stationary value. In both cases, in more elaborate experiments, another deformation or flow is given before the sample reaches equilibrium. Internal reorganization of chains in the network can be studied by such transient experiments.

\section{Stress Relaxation}

Let us first study time evolution of the stress after a sudden deformation is given to an equilibrium network. The deformation, being followed by constant strain $\hat{\lambda}(t)=\hat{\lambda} \theta(t)$, creates a stress which gradually relaxes with time. Long time behavior of stress relaxation following a large stepwise deformation is frequently measured in rheological experiments and known as the nonlinear stress relaxation.

It turns out that the nonlinear stress relaxation function $\Sigma_{x y}(t, \hat{\lambda})$ is not factorized into the product of a function of the time and a function of the deformation tensor, thus time-strain separability loses its physical background.

\section{Stress Overshoot}

In another important transient experiment, the rheometer is started from time $t=0$ at a constant shear rate $\dot{\gamma}$ (or other deformation rate such as elongational rate $\dot{\epsilon}$ ), and the time dependent shear and normal stresses (or elongational stress) are measured. At high 


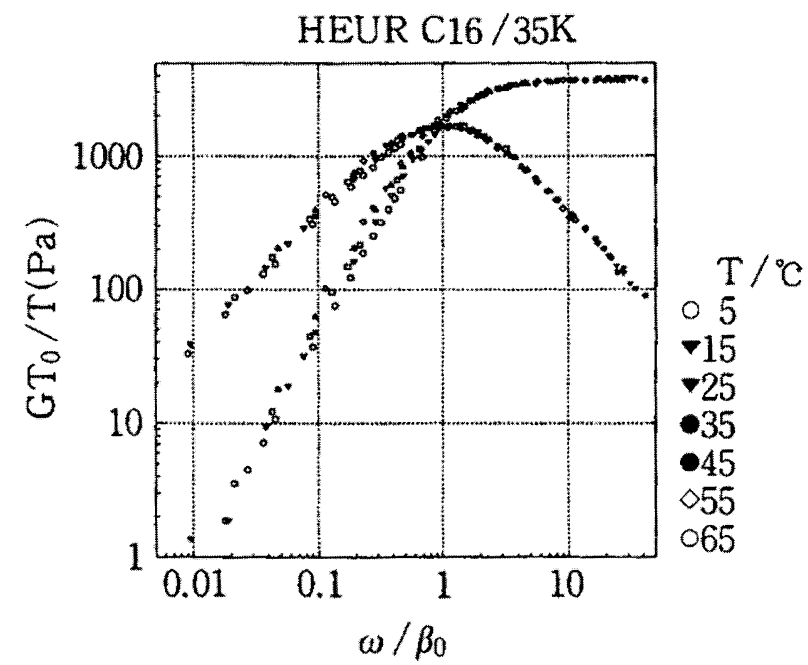

Figure 19. Viscoelastic master curve of HEUR with molecular weight $35 \mathrm{~K}$ and 16 Carbons in the end chain. The reference temperature is $5^{\circ} \mathrm{C}$. From the horizontal shift factor, the activation energy is found to be $67 \mathrm{~kJ} \mathrm{~mol}^{-1}$. From the high frequency plateau of the storage modulus, the number of elastically effective chains is found as a function of the polymer concentration.

shear rate, the stress exhibits a maximum in a certain transient region before reaching the stationary value. Such stress overshoot phenomena are commonly observed in polymer solutions and melts. Two important parameters are usually introduced for the characterization of the overshoot phenomena: the time $t^{*}$ at which the stress reaches the peak value (or equivalently, the amount of strain $\gamma^{*}=\dot{\gamma} t^{*}$ at this time), and the peak value of the stress $\Sigma\left(t^{*}\right)$ relative to its stationary value $\Sigma\left(t^{*}\right) / \Sigma(\infty)$. We now consider the time evolution of our transient network after a sudden steady flow is started.

In order to find the stress components, we must first solve the eq 99 for the number of active chains. Upon substitution of the solution into eq 102, time development of the stress tensor is found.

Let us first examine the GT limit. In this special case, we immediately find $v_{\text {in }}(t)=v_{0} e^{-\beta_{0} t}$ again as in the nonlinear stress relaxation, but we have $v(t)=v_{0}$. The total number of active chains stays constant in this limit even if a steady flow is started. It is easy to see that stresses are steadily increasing functions and exhibit no overshoot no matter how large the shear rate $\dot{\gamma}$ may become. In other words, if the chain breakage (end disengagement of a chain from a junction in our present model) takes place at a constant rate irrespective of the chain end-to-end distance, there should be no overshoot. The coupling between the two, i.e., the higher probability of breakage for more stretched chains, brings the stress overshoot. To see this in more detail, let us consider the quadratic breakage rate for which analytic solution can be found.

Figure 20 shows three components of the stress ten-

\section{STRESS \\ NR CHAINS}

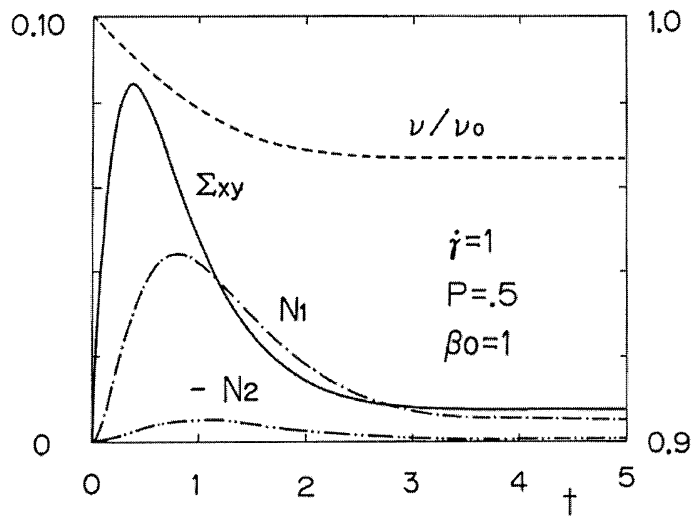

Figure 20. The stress overshoot of the transient network model under shear flow. The total number of active chains, the shear stress, the first- and second normal stress difference are shown as functions of time after a shear flow with the shear rate $\dot{\gamma}=1$ is started. The decay rate is fixed as $\beta_{0}=1$. Each component of the stress shows an overshoot at different time.

sor, together with the number of active chains, plotted against time. We assumed $\beta(r)=\beta_{0}+\beta_{1} r^{2} / 2$. The unit of the time is chosen as $\beta_{1}^{-1}$. The breakage rate $\beta_{0}$ and the probability $p$ of the end association are fixed as $\beta_{0}=1$ and $p=0.5$ relative to the value of $\beta_{1}$. The stationary value of the active chains is given by $v_{0} / n=0.19493$ in this case. It is evident that all stresses of our concern exhibit overshoot around the time at which the number of active chains decreases appreciably. The overshoot time differs for three stresses; the shear stress first shows its peak at $t_{x y}^{*}$, followed by a peak of the first normal stress difference at $t_{1}^{*}$, and then by the absolute value of the second normal stress difference at $t_{2}^{*}$. Notice that there is a large overshoot ratio of the shear stress.

\section{CONCLUSIONS}

We have presented an outline of our recent theoretical studies on molecular association and thermoreversible gelation in polymer solutions. Thermodynamic nature of the sol/gel transition, interference with phase separation, structure of the network junctions, path connectivity in the network have been studied on the basis of the multiple tree statistics combined with classical lattice-theoretical polymer solutions. Our studies have mainly focused on the gelation of watersoluble associating polymers driven by hydrophobic aggregation, but find many application to experimental data on other types of gels such as those driven by hydrogen bonding, micro-crystallization etc.

An attempt has also been made to find the linear and non-linear viscoelastic properties of reversibly cross-linked networks with a finite junction dissoci- 
ation time. The theoretical model proposed in this study approaches two extreme limits of the covalently crosslinked network and the uncrosslinked melt. The activation barrier for a chain dissociation and the number of elastically effective chains in the network are theoretically estimated and compared with the experiments. Transient flow properties such as stress relaxation and stress overshoot are derived. This study provides a possible pathway to modelling new types of transient networks such as hydrogen-bonded liquidcrystalline networks.

Acknowledgment. The author would like to thank Prof. Y. Morishima, Editor-in-Chief, for inviting this review article. He also thanks Dr. Ishida for his help in numerical calculation of the phase diagrams.

\section{REFERENCES}

1. A. H. Clark and S. B. Ross-Murphy, Adv. Polym. Sci., 83, 57 (1987).

2. P. S. Russo, "Reversible Polymeric Gels and Related Systems", ACS Symposium Series, No. 350, Am. Chem. Soc., Washington, D.C., 1987.

3. O. Kramer, "Biological and Synthetic Polymer Networks", Elsevier Applied Science, London and New York, 1988.

4. J. M. Guenet, "Thermoreversible Gelation of Polymers and Biopolymers", Academic Press, Inc. Harcourt Brace Jovanovich Publishers, 1992.

5. K. te Nijenhuis, $A d v$. Polym. Sci., 130, 1 (1997).

6. P. J. Flory, J. Chem. Phys., 12, 425 (1944).

7. M. L. Huggins, J. Chem. Phys., 46, 151 (1942).

8. P. J. Flory, "Principles of Polymer Chemistry", Cornell University Press, Ithaca, N.Y., (1953).

9. R. Koningsveld, W. H. Stockmayer, and E. Nies, "Polymer Phase Diagrams", Oxford University Press, Oxford, London, 2001.

10. F. Tanaka, Macromolecules, 22, 1988 (1989).

11. F. Tanaka and A. Matsuyama, Phys. Rev. Lett., 62, 2759 (1989).

12. F. Tanaka, Macromolecules, 23, 3784 (1990).

F. Tanaka, Macromolecules, 23, 3790 (1990).

13. F. Tanaka and T. Koga, Bull. Chem. Soc. Jpn, 74, 201 (2001).

14. K. Šolc, Macromolecules, 3, 665 (1970).

15. F. S. Bates and G. H. Fredrickson, Annu. Rev. Phys. Chem., 41, 525 (1990).

16. L. Leibler, Macromolecules, 13, 1602 (1980).

17. F. Tanaka, M. Ishida, and A. Matsuyama, Macromolecules, 24, 5582 (1991).

18. F. Tanaka and M. Ishida, Macromolecules, 30, 1836 (1997).

19. F. Tanaka, Phys. Rev. Lett., 68, 3188 (1992).

20. F. Tanaka and M. Ishida, Macromolecules, 32, 1271 (1999).

21. A. R. Shultz and P. J. Flory, J. Am. Chem. Soc., 75, 3888 (1953).

A. R. Shultz and P. J. Flory, J. Am. Chem. Soc., 75, 5631 (1953).

22. L. Kielhorn and M. Muthukumar, J. Chem. Phys., 107, 5588
(1997).

23. H. Tanaka and T. Hashimoto, Macromolecules, 24, 5398 (1991).

H. Tanaka and T. Hashimoto, Macromolecules, 24, 5713 (1991).

24. R. M. Hornreich, M. Luban, and S. Shtrikman, Phys. Rev. Lett., 35, 1678 (1975).

25. J.-M. Lehn, "Supramolecular Chemistry - Concepts and Perspectives", VCH Rublishers, New York, N.Y., 1995.

26. C. Ciferri, "Supramolecular Polymers", Marcel Dekker, Inc., New York, N.Y., 2000.

27. T. Kato, Struct. Bond., 96, 95 (2000).

28. J. Ruokolainen, G. ten Brinke, O. Ikkala, M. Torkkeli, and R. Serimaa, Macromolecules, 29, 3409 (1996).

29. J. Ruokolainen, M. Torkkeli, R. Serimaa, S. Vahvaselkä, M. Saariaho, G. ten Brinke, and O. Ikkala, Macromolecules, 29, 6621 (1996).

30. G. ten Brinke, J. Ruokolainen, and O. Ikkala, Europhys. Lett., 35, 91 (1996).

31. G. ten Brinke and O. Ikkala, Trends Polym. Sci., 5, 213 (1997).

32. J. Ruokolainen, M. Torkkeli, R. Serimaa, B. E. Komanschek, O. Ikkala, and G. ten Brinke, Phys. Rev. E: Stat. Phys., Plasmas, Fluids. Relat., 54, 6646 (1996).

33. A. Matsuyama and F. Tanaka, Phys. Rev. Lett., 65, 341 (1990).

34. G. R. Anderson and J. C. Wheeler, J. Chem. Phys., 73, 5778 (1980).

35. J. S. Walker and C. A. Vause, Phys. Lett. A, 79, 421 (1980).

36. R. E. Goldstein and J. S. Walker, J. Chem. Phys., 78, 1942 (1983).

37. J. S. Walker and C. A. Vause, Sci. Am., 256, 90 (1987).

38. T. Narayanan and A. Kumar, Phys. Rep., 249, 135 (1994).

39. S. Saeki, N. Kuwahara, S. Konno, and M. Kaneko, Macromolecules, 6, 247 (1973).

S. Saeki, N. Kuwahara, S. Konno, and M. Kaneko, Macromolecules, 8, 799 (1975).

40. S. Saeki, N. Kuwahara, M. Nakata, and M. Kaneko, Polymer, 17, 685 (1976).

41. A. E. Bradfield and B. Jones, J. Chem. Soc., 2660 (1929).

42. B. Jones, J. Chem. Soc., 1874 (1935).

43. C. Weygand and R. Gabler, Z. Phys. Chem. B, 46, 270 (1940).

44. G. W. Gray and B. Jones, J. Chem. Soc., 4179 (1953).

45. M.-J. Brienne, J. Gabard, J.-M. Lehnand, and I. Stibor, J. Chem. Soc. Chem. Comm., 1868 (1989).

46. T. Kato and J. M. J. Fréchet, Macromolecules, 22, 3818 (1989).

47. C. G. Bazuin, in "Mechanical and Thermophysical Properties of Polymer Liquid Crystals", W. Brostow, Ed., Chapman and Hall. Ltd., London, 1998, chapt. 3.

48. W. Maier and A. Saupe, Z. Naturforsch. A: Phys. Sci., 13, 564 (1958).

W. Maier and A. Saupe, Z. Naturforsch.A: Phys. Sci., 14, 882 (1959).

W. Maier and A. Saupe, Z. Naturforsch. A: Phys. Sci., 15, 287(1960).

49. W. L. McMillan, Phys. Rev. A: At., Mol., Opt. Phys., 4, 1238 (1971).

50. P. J. Flory, Proc. R. Soc., London Ser. A, 234, 73 (1956).

51. W. G. Miller, L. Kou, K. Tohyama and V. Voltaggio, J. Polym, Sci.: Polym. Symp., 65, 91 (1978). 
52. E. T. Whittaker and G. N. Watson, "A Course of Modern Analysis", Cambridge Univ. Press, Cambirdge, 1969.

53. C. Tanford, "The Hydrophobic Effects", John Wiley \& Sons, Inc., New York, N.Y., 1980.

54. R. L. Scott, J. Phys. Chem., 69, 261 (1965).

R. L. Scott, J. Phys. Chem., 71, 352 (1967).

55. S. J. Candau, E. Hirsch, and R. Zana, J. Phys. France, 45, 1263 (1984).

56. T. Shikata, H. Hirata, and T. Kotaka, Langmuir, 3, 1081 (1987).

T. Shikata, H. Hirata, and T. Kotaka, Langmuir, 4, 354 (1988).

57. H. Hofmann and H. Rehage, Mol. Phys., 5, 1225 (1989).

58. P. J. Flory, J. Am. Chem. Soc., 63, 3091 (1941).

P. J. Flory, J. Am. Chem. Soc., 63, 3096 (1941).

59. W. H. Stockmayer, J. Chem. Phys., 11, 45 (1943).

W. H. Stockmayer, J. Chem. Phys., 12, 125 (1944).

60. M. Ishida and F. Tanaka, Macromolecules, 30, 3900 (1997).

61. C. M. Knobler and R. L. Scott, in "Phase Transitions and Critical Phenomena”, Academic Press, Inc., New York, N.Y., 1984, vol. 9.

62. R. Pynn and A. Skjeltorp, "Multicritical Phenomena", Plenum Press, New York, N.Y., 1984.

63. S. Wellinghoff, J. Shaw, and E. Baer, Macromolecules, 12, 932 (1979).

64. H. M. Tan, A. Moet, A. Hiltnet, and E. Baer, Macromolecules, 16, 28 (1983).

65. R. F. Boyer, E. Baer, and A. Hiltner, Macromolecules, 18, 427 (1985).

66. R. C. Domszy, R. Alamo, C. O. Edwards, and L. Mandelkern, Macromolecules, 19, 310 (1986).

67. J. Y. S. Gan, J. Francois, and J. M. Guenet, Macromolecules, 19, 173 (1986).

68. S.-J. Chen, G. C. Berry, and D. J. Plazek, Macromolecules, 28, 6539 (1995)

69. M. A. Winnik, Curr. Opin. Colloid Interface Sci., 2, 424 (1997).

70. T. Annable, R. Buscall, R. Ettelaie, and D. Whittlestone, J. Rheol., 37, 695 (1993).

71. T. Annable, R. Buscall, R. Ettelaie, P. Shepherd, and D. Whittlestone, Langmuir, 10, 1060 (1994).

72. B. Rao, Y. Uemura, L. Dyke, and P. M. Macdonald, Macromolecules, 28, 531 (1995).

73. E. Alami, M. Almgren, W. Brown, and J. Francois, Macromolecules, 29, 2229 (1996).

74. E. Alami, M. Almgren, and W. Brown, Macromolecules, 29, 5026 (1996).

75. B. Xu, A. Yekta, and M. A. Winnik, Langmuir, 13, 6903 (1997).

76. K. C. Tam, R. D. Jenkins, M. A. Winnik, and D. R. Bassett, Macromolecules, 31, 4149 (1998).

77. Z. Zhou, Y.-W. Yang, C. Booth, and B. Chu, Macromolecules, 29, 8357 (1996)

78. E. J. Amis, N. Hu, T. A. P. Seery, T. E. Hogen-Esch, M. Yassini, and F. Hwang, in "Hydrophobic Polymers: Performance with Environmental Acceptance”, J. E. Glass, Ed., Advances in Chemistry Series, Am. Chem. Soc., Washington, D. C., 1996, p 279.

79. F. S. Hwang and T. E. Hogen-Esch, Macromolecules, 28, 3328 (1995).
80. X. Xie and T. E. Hogen-Esch, Macromolecules, 29, 1734 (1996).

81. H. Zhang, J. Pan, and T. E. Hogen-Esch, Macromolecules, 31, 2815 (1998).

82. S. Nilsson, Macromolecules, 28, 7837 (1995).

83. B. Nyström, K. Thuresson, and B. Lindman, Langmuir, 11, 1994 (1995).

84. B. Nyström, H. Walderhaug, F. K. Hansen, and B. Lindman, Langmuir, 11, 750 (1995).

85. N. Sarkar, J. Appl. Polym. Sci., 24, 1073 (1979).

86. K. Kobayashi, C. Huang, and T. P. Lodge, Macromolecules, 32, 7070 (1999).

87. Y. Morishima, Trends Polym. Sci., 2, 31 (1994).

88. Y. Morishima, S. Nomura, T. Ikeda, M. Seki, and M. Kamachi, Macromolecules, 28, 2874 (1995).

89. F. Petit, I. Iliopoulos, R. Audebert, and S. Szonyi, Langmuir, 13, 4229 (1997).

90. G. Bokias, D. Hourdet, I. Iliopoulos, G. Staikos, and R. Audebert, Macromolecules, 30, 8293 (1997).

91. G. Bokias, D. Hourdet, and I. Iliopoulos, Macromolecules, 33, 2929 (2000).

92. K. Fukui and T. Yamabe, Bull. Chem. Soc. Jpn, 40, 2052 (1967).

93. F. Tanaka and W. H. Stockmayer, Macromolecules, 27, 3943 (1994).

94. F. Tanaka and T. Koga, Comp. Theor. Polym. Sci., 10, 259 (2000).

95. F. Tanaka and M. Ishida, Macromolecules, 29, 7571 (1996).

96. J. E. Eldridge and J. D. Ferry, J. Phys. Chem., 58, 992 (1954).

97. F. Tanaka and K. Nishinari, Macromolecules, 29, 3625 (1996).

98. K. Nishinari and F. Tanaka, J. Chim. Phys., 93, 880 (1996).

99. P. J. Flory, Proc. R. Soc. London, Ser. A, 351, 351 (1976).

100. J. Scanlan, J. Polym. Sci., 43, 501 (1960).

101. L. C. Case, J. Polym. Sci., 45, 397 (1960).

102. D. S. Pearson and W. W. Graessley, Macromolecules, 11, 528 (1978).

103. D. Stauffer, "Introduction to Percolation Theory", chapt. 2, Taylor \& Francis, London, 1985.

104. S. Newman, W. R. Krigbaum, and D. K. Carpenter, J. Phys. Chem., 60, 648 (1956).

105. T. Kondo, C. Sawatari, R. S. J. Manley, and D. G. Gray, Macromolecules, 27, 210 (1994).

106. F. Tanaka and M. Ishida, J. Chem. Soc., Faraday Trans., 91, 2663 (1995).

107. T. P. Stossel, Sci. Am., 265, 40 (1994).

108. A. H. Clark, R. K. Richardson, S. Ross-Murphy, and J. M. Stubbs, Macromolecules, 16, 1367 (1983).

109. K. Nishinari and E. Doi, "Food Hydrocolloids: Structures, Properties, and Functions", Plenum Press, New York, N.Y., 1994.

110. L. H. Sperling, "Interpenetrating Polymer Networks and Related Materials", Plenum Press, New York, N.Y., 1981.

111. L. H. Sperling, "Multiphase Polymers: Blends and Ionomers", ACS Symposium Series No. 395, Am. Chem. Soc., Washington, D.C., 1988.

112. C. M. Durrani, D. A. Prystupa, A. M. Donald, and A. H. Clark, Macromolecules, 26, 981 (1993).

113. C. M. Durrani and A. M. Donald, Macromolecules, 27, 110 
(1994).

114. G. Bokias, D. Hourdet, I. Iliopoulos, G. Staikos, and R. Audebert, Macromolecules, 30, 8293 (1997).

115. W. H. Stockmayer, J. Polym. Sci., 9,69 (1952).

116. J. Francois, J. Y. S. Gan, and J. M. Guenet, Macromolecules, 19, 2755 (1986).

117. F. Tanaka, Macromolecules, 33, 4249 (2000).

118. V. B. Tolstoguzov, "Food Hydrocolloids: Structures, Properties, and Functions", Plenum Press, New York, N.Y., 1994, pp. 327-340.

119. P. G. Higgs and R. C. Ball, J. Phys. France, 50, 3285 (1989).

120. C. Viebke, L. Piculell, and S. Nilsson, Macromolecules, 27, 4160 (1994).

121. M. Berghmans, S. Thijs, M. Cornette, H. Berghmans, F. C. De Schryver, P. Moldenaers, and J. Mewis, Macromolecules, 27, 7669 (1994)

122. K. Buyse, H. Berghmans, M. Bosco, and S. Paoletti, Macromolecules, 31, 9224 (1998).

123. P. L. Biagio and M. U. Palma, Biophys. J., 60, 508 (1991).

124. A. Tobitani and S. B. Ross-Murphy, Macromolecules, 30, 4845 (1997).

A. Tobitani and S. B. Ross-Murphy, Macromolecules, 30, 4855 (1997).

125. B. Xu, A. Yekta, and M. A. Winnik, Langmuir, 13, 6903 (1997).

126. D. Poland and H. A. Scheraga, "Theory of Helix-Coil Transitions in Biopolymers", Academic Press, Inc., New York, N.Y., 1970.

127. A. V. Tobolsky and A. Eisenberg, J. Am. Chem. Soc., 81, 780 (1959).

128. R. L. Scott, J. Phys. Chem., 69, 261 (1965).

R. L. Scott, J. Phys. Chem., 71, 352 (1967).

129. P. Dubin Ed., "Microdomains in Polymer Solutions", Plenum Press, New York N.Y., 1985.

130. E. D. Goddard and K. P. Ananthapadmanabhan, Ed., "Interactions of Surfactants with Polymers and Proteins", CRC Press, Boca Raton, FL, 1993.

131. B. J. Cabane, J. Phys. Chem., 81, 1639 (1977).

132. W. Brown, J. Fundin, and M. da Graça Miguel, Macromolecules, 25, 7192 (1992).

133. E. Feitosa, W. Brown, and P. Hansson, Macromolecules, 29, 2169 (1996)
134. E. Feitosa, W. Brown, M. Vasilescu, and M. SwasonVethamuthu, Macromolecules, 29, 6837 (1996).

135. J. Rička, M. Meewes, R. Nyffenegger, and T. Binkert, Phys. Rev. Lett., 65, 657 (1990).

136. M. Meewes, J. Ricka, M. de Silva, R. Nyffenegger, and T. Binkert, Macromolecules, 24, 5811 (1991).

137. G. H. Fredrickson, Macromolecules, 26, 2825 (1993).

138. T. Seki, A. Tohnai, T. Tamaki, and A. Kaito, Macromolecules, 29, 4813 (1996).

139. E. Alami, M. Almgren, W. Brown, and J. Francois, Macromolecules, 29, 2229 (1996).

140. B. Cabane, K. Lindell, S. Engstrom, and B. Lindman, Macromolecules, 29, 3188 (1996).

141. B. Nyström, H. Walderhaug, F. K. Hansen, and B. Lindman, Langmuir, 11, 750 (1995).

142. B. Nyström, A. L. Kjonisken, and B. Lindman, Langmuir 12, 3233 (1996).

143. G. Wang, K. Lindell, and G. Olofsson, Macromolecules, 30, 105 (1997).

144. F. Tanaka, Macromolecules, 31, 384 (1998).

145. M. S. Green and A. V. Tobolsky, J. Chem. Phys., 14, 80 (1946).

146. A. S. Lodge, Trans. Faraday Soc., 52, 120 (1956).

147. M. Yamamoto, J. Phys. Soc. Jpn., 11, 413 (1956). M. Yamamoto, J. Phys. Soc. Jpn., 12, 1148 (1957). M. Yamamoto, J. Phys. Soc. Jpn., 11, 1200 (1958).

148. M. Doi and S. F. Edwards, "The Theory of Polymer Dynamics", Oxford University Press, London, 1986.

149. B. B. Bird, C. F. Curtiss, R. C. Armstrong, and O. Hassager, "Dynamics of Polymeric Liquids", John Wiley \& Sons, Inc, New York, N.Y., 1987, vol. 2, chapt. 20.

150. F. Tanaka and S. F. Edwards, Macromolecules, 25, 1516 (1992).

151. F. Tanaka and S. F. Edwards, J. Non-Newtonian Fluid Mech., 43, 247 (1992).

F. Tanaka and S. F. Edwards, J. Non-Newtonian Fluid Mech., 43, 272, (1992).

F. Tanaka and S. F. Edwards, J. Non-Newtonian Fluid Mech., 43, 289 (1992).

152. L. Leibler, M. Rubinstein, and R. H. Colby, Macromolecules, 24, 4701 (1991).

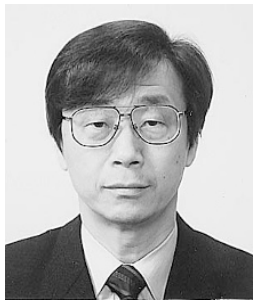

Fumihiko Tanaka was born in Nara in 1947. He graduated from the Department of Physics, Faculty of Science, The University of Tokyo in 1971, and received his Dr. of Science degree from The University of Tokyo in 1976. During his doctoral course research, he studied disordered magnets such as spin glasses and random magnetic fields. After working for two years and a half at the same department as a research associate, he moved to Cavendish Laboratory, Cambridge, UK, where he worked on the ground-state degeneracy of Edwards-Anderson spin glass model in collaboration with Sir Sam Edwards. In 1981, he joined the Department of Physics, Tokyo University of Agriculture and Technology as an Associate Professor, and became a Professor in 1994. His main research interests there were topological interaction in polymers including supercoiling of circular DNA, polymeric coil-globule transition and thermoreversible gelation. From 1990 to 1991, he spent another one year in Cambridge to work on the rheological properties of transient networks. He moved to Kyoto University in 1997. His research mainly applies various theoretical ideas and techniques to a variety of different problems in polymer science in close relation to experiments. He received The Award of the Scciety of Plolymer Science, Japan (2000). 